UDC $517.9+531.19$

D. Ya. Petrina (Inst. Math. Nat. Acad. Sci. Ukraine, Kyiv)

\title{
SPECTRUM AND STATES OF THE BCS HAMILTONIAN WITH SOURCES
}

\section{СПЕКТР ТА СТАНИ БКШ ГАМІЛЬТОНІАНА 3 ДЖЕРЕЛАМИ}

We consider the BCS Hamiltonian with the sources as it has been proposed by Bogolyubov and Bogolyubov (jr.). We prove that the eigenvectors and eigenvalues of the BCS Hamiltonian with the sources can be determined exactly in the thermodynamic limit. Earlier, Bogolyubov proved that the energies per volume of the BCS Hamiltonian with sources and the approximating Hamiltonian coincide in the thermodynamic limit.

Розглянуто БКШ гамільтоніан з джерелами, який був запропонований Боголюбовим та Боголюбовим (мол.). Доведено, що власні вектори та власні значення БКШ гамільтоніана 3 джерелами можна визначити точно в термодинамічній границі. Раніше Боголюбовим було встановлено, що питомі енергії БКШ та апроксимуючого гамільтоніанів збігаються в термодинамічній границі.

Introduction. In the series of papers [1-7] and book [8], we have investigated the spectrum and eigenfunctions of the BCS Hamiltonian [9]

$$
H_{\Lambda}=\sum_{\bar{p}}\left(\frac{p^{2}}{2 m}-\mu\right) a_{\bar{p}}^{+} a_{\bar{p}}+\frac{g}{V} \sum_{p_{1}, p_{2}} v_{p_{1}} v_{p_{2}} a_{p_{1}}^{+} a_{-p_{1}}^{+} a_{-p_{2}} a_{p_{2}}
$$

in a finite cube $\Lambda$ with periodic boundary conditions. Here, $p$ is discrete momenta $p=$ $=\frac{2 \pi}{L}\left(n_{1}, n_{2}, n_{3}\right), n_{i} \subset Z, i=1,2,3$. By $\bar{p}$ we denote momenta $p$ and spin $\sigma= \pm \frac{1}{2}$, $p=\left(p,+\frac{1}{2}\right),-p=\left(-p,-\frac{1}{2}\right), g$ is a coupling constant, $v_{p}$ is the potential, $\mu$ is the chemical potential, $V=L^{3}$ is the volume of the cube $\Lambda$, and $L$ is the length of the edge of the cube.

In the special subspace of pairs we have represented the Hamiltonian $H_{\Lambda}$ as sum of two operators $\mathcal{A}_{\Lambda}$ and $\mathcal{B}_{\Lambda}$. The spectrum and eigenfunctions of the operator $\mathcal{A}_{\Lambda}$ can be determined exactly in subspaces of arbitrary $n$ pairs, and pairs do not interact. The operator $\mathcal{B}_{\Lambda}$ describes the interaction of pairs and it tends to zero as the volume $V$ tends to infinity for an arbitrary finite number $n$ of pairs. The operator $\mathcal{B}_{\Lambda}$ can be considered as a perturbation of the operator $\mathcal{A}_{\Lambda}$, and the spectrum of the operator $H_{\Lambda}$ is a perturbation of the spectrum of the operator $\mathcal{A}_{\Lambda}$ and can be determined asymptotically exactly as the volume $V$ tends to infinity.

It is not a surprise that the Hamiltonian $H_{\Lambda}$ has eigenfunctions in the subspace of $n$ pairs because the operator of the number of particles commutes with the Hamiltonian $H_{\Lambda}$. It is a great surprise that this phenomenon has not been recognized earlier.

We have discovered a new branch of the spectrum and eigenfunctions of the BCS Hamiltonian $H_{\Lambda}$ that differs from the well-known spectrum corresponding to the BCS ground state and its excitations. This new branch of eigenfunctions consists of an arbitrary number $n=1,2, \ldots$ of pairs in ground state and excitation of these pairs with an arbitrary orbital momenta $l=0,1, \ldots$ and a continuous energy divided by a nonzero gap from the energy of the pairs in ground state. 
From the physical point of view, the first main difference between the classical BCS branch of spectrum and eigenfunctions and the new branch of spectrum and eigenfunctions consists of the following: In the excitations of the BCS ground states, a certain number of pairs are replaced by the same number of electrons. In the excitations of the new ground state, a certain number of pairs are replaced by the same number of pairs with orbital momenta and continuous energy divided from the energy of pairs in the ground state by a nonzero gap.

The same situation is true for the excitations. Namely, an arbitrary number $n$ of excited pairs of the second branch is the eigenfunction of the BCS Hamiltonian, while excited $n$ pairs of the first branch are not eigenfunction of the BCS Hamiltonian.

The second main difference consists of the following: the BCS ground state is a coherent vector of the same pairs, as well as the ground state of the new branch. But each pair and an arbitrary number of pairs of the new branch are eigenvectors of the BCS Hamiltonian in the thermodynamic limit when an arbitrary number of pairs of the BCS ground state are not eigenfunctions of the BCS Hamiltonian. It is interesting to mention that Cooper in his famous paper [10], in fact, investigated the BCS Hamiltonian in the subspace of one pair and promised to investigate the spectrum in the subspace of an arbitrary number $n$ of pairs. We solved this problem in the series of our papers $[1-7]$ and the book [8].

In the given paper, we consider the BCS Hamiltonian with sources, as it has been proposed by Bogolyubov [11] and Bogolyubov(jr.) [12]. Namely, we consider the Hamiltonian

$$
\begin{gathered}
H_{\Lambda, \nu}=\sum_{\bar{p}}\left(\frac{p^{2}}{2 m}-\mu\right) a_{\bar{p}}^{+} a_{\bar{p}}+\frac{g}{V} \sum_{p_{1}, p_{2}} v_{p_{1}} v_{p_{2}} a_{p_{1}}^{+} a_{-p_{1}}^{+} a_{-p_{2}} a_{p_{2}}+ \\
+\nu \sum_{p} v_{p} a_{p}^{+} a_{-p}^{+}+\nu \sum_{p} v_{p} a_{-p} a_{p}
\end{gathered}
$$
where $\nu>0$ is parameter and $\nu \sum_{p} v_{p} a_{p}^{+} a_{-p}^{+}$and $\nu \sum_{p} v_{p} a_{-p} a_{p}$ are the operators of
sources.

Bogolyubov had explained [13] that sources are introduced into the original BCS Hamiltonian in order to choose the proper solution (eigenvectors). In the final results one should put $\nu=0$. It will be shown that the Hamiltonian with sources (2) has only one branch of the spectrum, namely the ground state and its excitations discovered by Bardeen, Cooper, and Schrieffer. The eigenvectors and their eigenvalues depend continuously on the parameter $\nu$, but at $\nu=0$ they coincide with the corresponding BCS eigenvectors and their eigenvalues, but not with the second branch. This means that the BCS Hamiltonian remembers perturbation by sources, which are unbounded operators.

The BCS Hamiltonian with sources does not have eigenfunctions in the subspace of $n$ pairs because the operators of sources connect the subspaces of $n+1$ and $n-1$ pairs with the subspace of $n$ pairs. Therefore, it is necessary to consider the eigenvalue problem in the entire subspace of pairs. We showed that the BCS Hamiltonian with sources (2) can be represented in it as the sum of two operators $\mathcal{A}_{\Lambda, \nu}$ and $\mathcal{B}_{\Lambda}$, where the operator $B_{\Lambda}$ tends to zero in the thermodynamic limit and can be considered as a pertur- 
bation. The operator $\mathcal{A}_{\Lambda, \nu}$ coincides in the thermodynamic limit with the approximating Hamiltonian on the ground and excited BCS states

$$
\begin{aligned}
H_{\Lambda, \nu}^{a}= & \sum_{\bar{p}}\left(\frac{p^{2}}{2 m}-\mu\right) a_{\bar{p}}^{+} a_{\bar{p}}+\left(c_{\Lambda}+\nu\right) \sum_{p} v_{p} a_{p}^{+} a_{-p}^{+}+ \\
& +\left(c_{\Lambda}+\nu\right) \sum_{p} v_{p} a_{p}^{+} a_{-p} a_{p}-g^{-1} c_{v}^{2} V I,
\end{aligned}
$$

where $c_{\Lambda}$ is a certain constant and $I$ is the identity operator. As is known, the spectrum and eigenvectors of the approximating Hamiltonian can be determined exactly.

Thus, we have proved that the eigenvectors and eigenvalues of the BCS Hamiltonian can be determined exactly in the thermodynamics limit. Earlier, Bogolyubov [11] proved that the energies per volume of the BCS Hamiltonian with sources (2) and of the approximating Hamiltonian coincide in the thermodynamic limit. His result was very unique information about the spectrum of the BCS Hamiltonian.

1. BCS Hamiltonian with sources in the subspace of pairs. 1.1. Action of the BCS Hamiltonian with sources in the subspace of pairs. Consider the BCS Hamiltonian with sources in a finite cube $\Lambda$ of volume $V$

$$
\begin{gathered}
H_{\Lambda, \nu}=\sum_{\bar{p}}\left(\frac{p^{2}}{2 m}-\mu\right) a_{\bar{p}}^{+} a_{\bar{p}}+\frac{g}{V} \sum_{p_{1}, p_{2}} v_{p_{1}} v_{p_{2}} a_{p_{1}}^{+} a_{-p_{1}}^{+} a_{-p_{2}} a_{p_{2}}+ \\
+\nu \sum_{p} v_{p} a_{p}^{+} a_{-p}^{+}+\nu \sum_{p} v_{p} a_{-p} a_{p},
\end{gathered}
$$

where $\nu>0$ is the same parameter, which characterizes the last two operators in $H_{\Lambda, \nu}$ known as sources. Note that the summation is carried out over momenta $p=$ $=\frac{2 \pi}{4}\left(n_{1}, n_{2}, n_{3}\right)$, where $L$ is the length of the edge of the cube $\Lambda$ and $n_{i}$ are integer numbers $i=1,2,3, \bar{p}=(p, \pm 1)$. The original BCS Hamiltonian is obtained for $\nu=0$, i.e., $\left.H_{\Lambda, \nu}\right|_{\nu=0}=H_{\Lambda}$.

The sources have been introduced by Bogolyubov [11] "in order to choose a proper solution for eigenvalue problem".

We introduce the subspace of pairs $\mathcal{H}^{P}$

$$
\begin{aligned}
f & =\sum_{n=0}^{\infty} \frac{1}{n !} \sum_{k_{1} \neq \ldots \neq k_{n}} f_{n}\left(k_{1}, \ldots, k_{n}\right) a_{k_{1}}^{+} a_{-k_{1}}^{+} \ldots a_{k_{n}}^{+} a_{-k_{n}}^{+}|0\rangle= \\
& =\sum_{n=0}^{\infty} \frac{1}{n !} \sum_{k_{1}, \ldots, k_{n}} f_{n}\left(k_{1}, \ldots, k_{n}\right) a_{k_{1}}^{+} a_{-k_{1}}^{+} \ldots a_{k_{n}}^{+} a_{-k_{n}}^{+}|0\rangle,
\end{aligned}
$$

where $f_{n}\left(k_{1}, \ldots, k_{n}\right)$ are the wave functions of $n$ pairs of electrons with opposite momenta and spins and are symmetric, $|0\rangle$ is the vacuum state, and the summation is carried out over all $k_{1} \neq \ldots \neq k_{n}$. We suppose that the functions $f_{n}\left(k_{1}, \ldots, k_{n}\right)$ are defined for all momenta $k_{1}, \ldots, k_{n}$, but not only for $k_{1} \neq \ldots \neq k_{n}$. In the last expression on the right-hand side of (1.2) we add terms with equal momenta. These terms are equal to zero.

In the subspace of pairs $\mathcal{H}^{P}$ we introduce the following scalar product of two elements $f$ and $g$ : 


$$
\begin{aligned}
(f, g)^{\prime} & =\sum_{n=0}^{\infty} \frac{1}{n !} \sum_{k_{1} \neq \ldots \neq k_{n}} \overline{f_{n}\left(k_{1}, \ldots, k_{n}\right)} g_{n}\left(k_{1}, \ldots, k_{n}\right)= \\
& =\sum_{n=0}^{\infty} \sum_{k_{1} \neq \ldots \neq k_{n}}^{\prime} \overline{f_{n}\left(k_{1}, \ldots, k_{n}\right)} g_{n}\left(k_{1}, \ldots, k_{n}\right),
\end{aligned}
$$

where $\sum_{k_{1} \neq \ldots \neq k_{n}}^{\prime}$ means that the summation is carried out over all $k_{1} \neq \ldots \neq k_{n}$, and the points $k_{1} \neq \ldots \neq \ldots k_{n}$ that differ only by permutations are identified. The norm is defined as follows: $\|f\|^{\prime}=\left\{(f, f)^{\prime}\right\}^{\frac{1}{2}}$.

We will also use the scalar product of two elements $f$ and $g$ defined as follows:

$$
(f, g)=\sum_{n=0}^{\infty} \frac{1}{n !} \sum_{k_{1}, \ldots, k_{n}} \overline{f_{n}\left(k_{1}, \ldots, k_{n}\right)} g_{n}\left(k_{1}, \ldots, k_{n}\right),
$$

where the summation is carried out over all $k_{1}, \ldots, k_{n}$, including momenta that coincide. We have the norm $\|f\|=(f, f)^{\frac{1}{2}}$.

In order to perform the thermodynamic limit we need the following scalar products and norms:

$$
\begin{gathered}
(f, g)_{V}^{\prime}=\sum_{n=0}^{\infty} \frac{1}{V^{n}} \sum_{k_{1} \neq \ldots \neq k_{n}}^{\prime} \frac{f_{n}\left(k_{1}, \ldots, k_{n}\right)}{g_{n}}\left(k_{1}, \ldots, k_{n}\right)= \\
=\sum_{n=0}^{\infty} \frac{1}{n !} \frac{1}{V^{n}} \sum_{k_{1} \neq \ldots \neq k_{n}} f_{n}\left(k_{1}, \ldots, k_{n}\right) g_{n}\left(k_{1}, \ldots, k_{n}\right), \\
\|f\|_{V}^{\prime}=\left\{(f, f)_{V}^{\prime}\right\}^{\frac{1}{2}}
\end{gathered}
$$

and

$$
\begin{gathered}
(f, g)_{V}=\sum_{n=0}^{\infty} \frac{1}{n !} \frac{1}{V^{n}} \sum_{k_{1}, \ldots, k_{n}} \overline{f_{n}\left(k_{1}, \ldots, k_{n}\right)} g_{n}\left(k_{1}, \ldots, k_{n}\right) \\
\|f\|_{V}=\left\{(f, f)_{V}\right\}^{\frac{1}{2}}
\end{gathered}
$$

We suppose that the potential $v_{p}$ satisfies the following conditions: it has support on compact $\mathcal{D}$, it is continuous for $p \in \mathcal{D}$ and $\frac{1}{V} \sum_{p}\left|v_{p}\right|^{2}=\|v\|^{2}<\infty$, where $\sup _{p \in \mathcal{D}}\left|v_{p}\right|=v<\infty$ uniformly with respect to $\Lambda(V)$. The wave functions of $n$ pairs $f_{n}\left(k_{1}, \ldots, k_{n}\right)$ are supposed to have support in the domain $\mathcal{D}$ with respect to all the momenta $k_{1}, \ldots, k_{n}$.

Repeating the calculation performed in our previous paper [6] with obvious modifications connected with sources, one obtains for $H_{\Lambda, \nu} f$ the following expression:

$$
\begin{gathered}
\left(H_{\Lambda, \nu} f\right)_{n}\left(k_{1}, \ldots, k_{n}\right)=\left(\frac{2 k_{1}^{2}}{2 m}+\ldots+\frac{2 k_{n}^{2}}{2 m}-2 \mu n\right) f_{n}\left(k_{1}, \ldots, k_{n}\right)+ \\
+\frac{g}{V} \sum_{i=1}^{n} v_{k_{i}} v_{p} f_{n}\left(k_{1}, \ldots, \frac{i}{p}, \ldots, k_{n}\right)+
\end{gathered}
$$




$$
\begin{gathered}
+\nu \sum_{i=1}^{n} v_{k_{i}} f_{n-1}\left(k_{1}, \ldots, \vee^{i}, \ldots, k_{n}\right)+\nu \sum_{p \neq(k)_{n}} v_{p} f_{n+1}\left(p, k_{1}, \ldots, k_{n}\right)- \\
-\frac{g}{V} \sum_{i=1}^{n} \sum_{1=j \neq i}^{n} v_{k_{i}} v_{k_{j}} f_{n}\left(k_{1}, \ldots, \frac{i}{k_{j}}, \ldots, k_{n}\right), \\
(k)_{n}=\left(k_{1}, \ldots, k_{n}\right), \quad n \geq 1,
\end{gathered}
$$

where $\left(k_{1}, \ldots, \frac{i}{p}, \ldots, k_{n}\right) \equiv\left(k_{1}, \ldots, k_{i-1}, p, k_{i+1} \ldots, k_{n}\right)$ and $\left(k_{1}, \ldots, \stackrel{i}{\vee}, \ldots, k_{n}\right) \equiv$ $\equiv\left(k_{1}, \ldots, k_{i-1}, k_{i+1}, \ldots, k_{n}\right)$.

Note that expression (1.7) was derived, according to the Fermi statistics, for all momenta such that $k_{1} \neq \ldots \neq k_{n}$. Due to the fact that functions $f_{n}\left(k_{1}, \ldots, k_{n}\right)$ are defined for all $k_{1}, \ldots, k_{n}$, but not only for $k_{1} \neq \ldots \neq k_{n}$, we will suppose that expression (1.7) is also true for all $k_{1}, \ldots, k_{n}$. For example, equating in expression $H_{\Lambda, \nu} f$ coefficients of the same products of the operators of creation pairs, including operators with equal momenta, one obtains expression (1.7) for all $k_{1}, \ldots, k_{n}$ including equal momenta. In what follows we will consider both expressions when we investigate the eigenvalue and eigenvector problem for the Hamiltonian $H_{\Lambda, \nu}$.

Note that both expressions coincide in the thermodynamic limit as $V \rightarrow \infty(\Lambda \nearrow$ $\nearrow \mathbb{R}^{3}$ ). This will be shown later in subsection 3.1.

Denote by $\mathcal{A}_{\Lambda, \nu}$ and $\mathcal{B}_{\Lambda}$ the following operators:

$$
\begin{gathered}
\left(\mathcal{A}_{\Lambda, \nu} f\right)_{n}\left(k_{1}, \ldots, k_{n}\right)=\left(\frac{2 k_{1}^{2}}{2 m}+\ldots+\frac{2 k_{n}^{2}}{2 m}-2 \mu n\right) f_{n}\left(k_{1}, \ldots, k_{n}\right)+ \\
+\frac{g}{V} \sum_{i=1}^{n} v_{k_{i}} v_{p} f_{n}\left(k_{1}, \ldots, \frac{i}{p}, \ldots, k_{n}\right)+ \\
+\nu \sum_{i=1}^{n} v_{k_{i}} f_{n-1}\left(k_{1}, \ldots, \stackrel{i}{\vee}, \ldots, k_{n}\right)+\nu \sum_{p \neq(k)_{n}} v_{p} f_{n+1}\left(p, k_{1}, \ldots, k_{n}\right), \\
\left(\mathcal{B}_{\Lambda} f\right)_{n}\left(k_{1}, \ldots, k_{n}\right)=-\frac{g}{V} \sum_{i=1}^{n} \sum_{1=j \neq i}^{n} v_{k_{i}} v_{k_{j}} f_{n}\left(k_{1}, \ldots, \frac{i}{k_{j}}, \ldots, k_{n}\right) .
\end{gathered}
$$

In what follows, we will consider the operators $\mathcal{A}_{\Lambda, \nu}$ and $\mathcal{B}_{\Lambda}$ defined by formulas (1.4), for $k_{1} \neq \ldots \neq k_{n}$ and as well for all $k_{1}, \ldots, k_{n}$.

Now we want to attract attention to some special properties of the operator $H_{\Lambda, V}$, namely, to the operator $\mathcal{A}_{\Lambda, \nu}$. If $\nu=0$, then the subspaces of $n$ pairs are invariant with respect to $H_{\Lambda}, \mathcal{A}_{\Lambda}=\mathcal{A}_{\Lambda, 0}$, and $\mathcal{B}_{\Lambda}$, and we have considered and investigated the eigenvalue problem in the subspaces. One can see from (1.8) that the subspaces of $n$ pairs are not invariant with respect to $H_{\Lambda, \nu}$ and $\mathcal{A}_{\Lambda, \nu}$ because $\left(\mathcal{A}_{\Lambda, \nu} f\right)_{n}\left(k_{1}, \ldots, k_{n}\right)$ contains terms with $f_{n-1}$ and $f_{n+1}$. Therefore, we can consider the eigenvalue problems for $H_{\Lambda, \nu}$ and $\mathcal{A}_{\Lambda, \nu}$ only in the entire subspace of pairs.

Prior to the investigation of this problem, we define the domain of definition of the Hamiltonian $H_{\Lambda, \nu}$ and the operators $\mathcal{A}_{\Lambda, \nu}$ and $\mathcal{B}_{\Lambda}$. If follows from formulas (3.3) and 
(3.4) of our paper [3] that the operator $\mathcal{A}_{\Lambda}=\mathcal{A}_{\Lambda, 0}$ is well defined and bounded in the subspaces $\mathcal{H}_{n}^{P}$ of $n$ pairs if the potential $v_{p_{1}} v_{p_{2}}$ satisfies the above described conditions. The operator $\mathcal{B}_{\Lambda}$, according to estimates $\left(3.9^{\prime}\right)$ and $\left(3.9^{\prime \prime}\right)$ in [3], is a bounded operator in $\mathcal{H}_{n}^{P}$ with scalar product (1.4), $\|B\| \leq \frac{g|v||v|}{V^{\frac{1}{2}}} n(n-1)$, and its norm tends to zero as $V \rightarrow \infty$ for arbitrary fixed $n$.

It is interesting to mention that Cooper in his famous paper [10], in fact, investigated the BCS Hamiltonian in the subspace of one pair and promised to investigate the spectrum in the subspace of an arbitrary number $n$ of pairs. We solved this problem in the series of our papers [1-7] and the book [8]. Thus, to define the operator $H_{\Lambda, \nu}$ it is sufficient to estimate the norms of the operators of sources.

1.2. Estimates of the norms of the operators of sources. Consider the operators of the sources $\nu \sum_{p} v_{p} a_{p}^{+} a_{-p}^{+}$, and $\nu \sum_{p} v_{p} a_{-p} a_{p}$. It follows from (1.7) that

$$
\begin{aligned}
\left(\nu \sum_{p} v_{p} a_{p}^{+} a_{-p}^{+} f\right)_{n}\left(k_{1}, \ldots, k_{n}\right) & =\nu \sum_{i=1}^{n} v_{k_{i}} f_{n-1}\left(k_{1}, \ldots, \stackrel{i}{\vee}, \ldots, k_{n}\right) \\
\left(\nu \sum_{p} v_{p} a_{-p} a_{p} f\right)_{n}\left(k_{1}, \ldots, k_{n}\right) & =\nu \sum_{p \neq(k)_{n}} v_{p} f_{n+1}\left(p, k_{1}, \ldots, k_{n}\right) \\
\nu & >0 .
\end{aligned}
$$

It is easy to obtain the following equalities:

$$
\begin{gathered}
\frac{1}{n !} \sum_{k_{1}, \ldots, k_{n}}\left|v_{k_{1}} f_{n-1}\left(k_{2}, \ldots, k_{n}\right)\right|^{2}= \\
=\frac{1}{n !} V \frac{1}{V} \sum_{k_{1}} v_{k_{1}^{2}} \sum_{k_{1}, \ldots, k_{n}}\left|f_{n-1}\left(k_{2}, \ldots, k_{n}\right)\right|^{2}=V \frac{1}{n}\|v\|^{2}\left\|f_{n-1}\right\|^{2}, \\
\frac{1}{n !} \sum_{k_{1}, \ldots, k_{n}}\left|\sum_{p \neq(k)_{n}} v_{p} f_{n+1}\left(p, k_{1}, \ldots, k_{n}\right)\right|^{2} \leq \\
\leq \frac{1}{n !} V \frac{1}{V} \sum_{p}\left|v_{p}\right|^{2} \sum_{p, k_{1}, \ldots, k_{n}}\left|f_{n+1}\left(p, k_{1}, \ldots, k_{n}\right)\right|^{2}=V(n+1)\|v\|^{2}\left\|f_{n+1}\right\|^{2} .
\end{gathered}
$$

Note that we have used the norm connected with the scalar product (1.4).

From these inequalities one gets

$$
\begin{gathered}
\left\|\nu\left(\sum_{p} v_{p} a_{p}^{+} a_{-p}^{+} f\right)_{n}\right\| \leq n^{\frac{1}{2}} V^{\frac{1}{2}} \nu\|v\|\left\|f_{n-1}\right\|, \\
\left\|\nu\left(\sum_{p} v_{p} a_{-p} a_{p} f\right)_{n}\right\| \leq(n+1)^{\frac{1}{2}} V^{\frac{1}{2}} \nu\|v\|\left\|f_{n+1}\right\|
\end{gathered}
$$


and this means that the sources are unbounded operators when $V \rightarrow \infty$ even in the subspace of $n$ pairs $\mathcal{H}_{n}^{P}$.

Finally, one has

$$
\begin{gathered}
\left\|\nu \sum_{p} v_{p} a_{p}^{+} a_{-p}^{+} f\right\| \leq V^{\frac{1}{2}} \nu\|v\| \sum_{n=0}^{\infty}(n+1)^{\frac{1}{2}}\left\|f_{n}\right\|, \\
\left\|\nu \sum_{p} v_{p} a_{-p} a_{p} f\right\| \leq V^{\frac{1}{2}} \nu\|v\| \sum_{n=0}^{\infty}(n+1)^{\frac{1}{2}}\left\|f_{n+1}\right\| .
\end{gathered}
$$

Note that summation with respect to $n$ is carried out from 0 to $N$, where $N$ is the number of quasimomenta $\frac{2 \pi}{L}\left(n_{1}, n_{2}, n_{3}\right)$ which lie in the domain $\mathcal{D}$ and $N$ tends to infinity as $V \rightarrow \infty$.

It follows from (1.10) that the operators of sources become unbounded in the thermodynamic limit as $V \rightarrow \infty$ in the whole subspace of pairs $\mathcal{H}^{P}$.

Now estimate the norms of the operators of sources using the norm of subspace of pairs (1.6) connected with the volume $V$. One has

$$
\begin{gathered}
\frac{1}{n !} \frac{1}{V^{n}} \sum_{k_{1}, \ldots, k_{n}}\left|v_{k_{1}} f_{n-1}\left(k_{2}, \ldots, k_{n}\right)\right|^{2}= \\
=\frac{1}{n !} \frac{1}{V} \sum_{k_{1}} v_{k_{1}^{2}} \frac{1}{V^{n-1}} \sum_{k_{1}, \ldots, k_{n}}\left|f_{n-1}\left(k_{2}, \ldots, k_{n}\right)\right|^{2}=\|v\|_{V}^{2} \frac{1}{n}\left\|f_{n-1}\right\|_{V}^{2}, \\
\frac{1}{n !} \frac{1}{V^{n}} \sum_{k_{1}, \ldots, k_{n}}\left|\sum_{p \neq\left(k_{n}\right)} v_{p} f_{n+1}\left(p, k_{1}, \ldots, k_{n}\right)\right|^{2} \leq \\
\leq \frac{1}{n !} V^{2} \frac{1}{V} \sum_{p} v_{p}^{2} \frac{1}{V^{n+1}} \sum_{p, k_{1}, \ldots, k_{n}}\left|f_{n+1}\left(p, k_{1}, \ldots, k_{n}\right)\right|^{2}= \\
=(n+1) V^{2}\|v\|_{V}^{2}\left(\left\|f_{n+1}\right\|_{V}\right)^{2} .
\end{gathered}
$$

From these inequalities one gets

$$
\begin{gathered}
\left\|\nu\left(\sum_{p} v_{p} a_{p}^{+} a_{-p}^{+} f\right)_{n}\right\|_{V} \leq n^{\frac{1}{2}} \nu\|v\|_{V}\left\|f_{n-1}\right\|_{V}, \\
\left\|\nu\left(\sum_{p} v_{p} a_{-p} a_{p} f\right)_{n}\right\|_{V} \leq(n+1)^{\frac{1}{2}} \nu V\|v\|_{V}\left\|f_{n+1}\right\|_{V} .
\end{gathered}
$$

Finally one has 


$$
\begin{gathered}
\left\|\nu \sum_{p} v_{p} a_{p}^{+} a_{-p}^{+} f\right\|_{V} \leq \nu\|v\|_{V} \sum_{n=0}^{\infty}(n+1)^{\frac{1}{2}}\left\|f_{n}\right\|_{V}, \\
\left\|\nu \sum_{p} v_{p} a_{-p} a_{p} f\right\|_{V} \leq V \nu\|v\|_{V} \sum_{n=0}^{\infty}(n+1)^{\frac{1}{2}}\left\|f_{n+1}\right\|_{V} .
\end{gathered}
$$

It follows from (1.11) that the operator of sources becomes unbounded as $V \rightarrow \infty$ because the volume $V$ is present in the second inequality in (1.11).

The unboundedness of the operators of sources has an important consequence. Namely, this means that even for arbitrary small parameter $\nu$ the operators of sources cannot be considered as the small perturbation of the operator $H_{\Lambda}=\left.H_{\Lambda, \nu}\right|_{\nu=0}$. We will show later that the eigenvalues and eigenvectors of the operators $H_{\Lambda, \nu}$, which will be calculated exactly in the thermodynamic limit (as $V \rightarrow \infty$ ), do not coincide when $\nu=0$ with the corresponding eigenvalues and eigenvectors of the operator $H_{\Lambda}($ as $V \rightarrow \infty)$.

2. Representation of $\boldsymbol{H}_{\Lambda, \nu}$ through the approximating Hamiltonian on coherent states of pairs. 2.1. Hamiltonian $\boldsymbol{H}_{\Lambda, \nu}$ on coherent states of pairs. Consider the state of pairs (1.2) with wave functions of $n$ pairs which are equal to the product of wave functions of one pair, namely

$$
\begin{aligned}
f= & \sum_{n=0}^{\infty} \frac{1}{n !} \sum_{k_{1} \neq \ldots \neq k_{n}} f_{1}\left(k_{1}\right) \ldots f_{1}\left(k_{n}\right) a_{k_{1}}^{+} a_{-k_{1}}^{+} \ldots a_{k_{n}}^{+} a_{-k_{n}}^{+}|0\rangle= \\
& =\sum_{n=0}^{\infty} \frac{1}{n !} \sum_{k_{1}} f_{1}\left(k_{1}\right) a_{k_{1}}^{+} a_{-k_{1}}^{+} \ldots \sum_{k_{n}} f_{1}\left(k_{n}\right) a_{k_{n}}^{+} a_{-k_{n}}^{+}|0\rangle .
\end{aligned}
$$

Then one has the following identity:

$$
\begin{gathered}
\frac{1}{n !} \sum_{k_{1} \neq \ldots \neq k_{n}} \frac{g}{V} \sum_{i=1}^{n} v_{k_{i}} \sum_{p} v_{p} f_{1}(p) f_{1}\left(k_{1}\right) \ldots \\
\ldots \stackrel{i}{\vee} \ldots f_{1}\left(k_{n}\right) a_{k_{1}}^{+} a_{-k_{1}}^{+} \ldots \stackrel{i}{\vee} \ldots a_{k_{n}}^{+} a_{-k_{n}}^{+}|0\rangle= \\
=\frac{c_{\Lambda}}{n !} \sum_{i=1}^{n} \sum_{k_{1} \neq \ldots \neq k_{n}} v_{k_{i}} f_{1}\left(k_{1}\right) \ldots \stackrel{i}{\vee} \ldots f_{1}\left(k_{n}\right) a_{k_{1}}^{+} a_{-k_{1}}^{+} \ldots \stackrel{i}{\vee} \ldots a_{k_{n}}^{+} a_{-k_{n}}^{+}|0\rangle= \\
=\frac{c_{\Lambda}}{n !} \sum_{i=1}^{n} \sum_{k_{1}, \ldots, k_{n}} v_{k_{i}} f_{1}\left(k_{1}\right) \ldots \vee^{i} \ldots f_{1}\left(k_{n}\right) a_{k_{1}}^{+} a_{-k_{1}}^{+} \ldots a_{k_{i}}^{+} a_{-k_{i}}^{+} \ldots a_{k_{n}}^{+} a_{-k_{n}}^{+}|0\rangle,
\end{gathered}
$$

where

$$
a_{k_{1}}^{+} a_{-k_{1}}^{+} \ldots \stackrel{i}{\vee} \ldots a_{k_{n}}^{+} a_{-k_{n}}^{+} \equiv a_{k_{1}}^{+} a_{-k_{1}}^{+} \ldots a_{k_{i-1}}^{+} a_{-k_{i-1}}^{+} a_{k_{i+1}}^{+} a_{-k_{i+1}}^{+} \ldots a_{k_{n}}^{+} a_{-k_{n}}^{+}
$$

and

$$
c_{\Lambda}=\frac{g}{V} \sum_{p} v_{p} f_{1}(p)
$$


Note that in (2.2) we added the terms with equal momenta which are equal to zero. Denote by $\mathcal{A}_{\Lambda}^{I}$ the part of the operator $\mathcal{A}_{\Lambda, \nu}$, that describes the interaction of two particles with opposite momenta and spin. It is defined by the second term in (1.7). It follows from (2.2) that the operator $\mathcal{A}_{\Lambda}^{I}$ on the state (2.1) is defined as follows:

$$
\begin{gathered}
\mathcal{A}_{\Lambda}^{I} f=c_{\Lambda} \sum_{n=1}^{\infty} \frac{1}{n !} \sum_{i=1}^{n} \sum_{k_{1} \neq \ldots \neq k_{n}} f\left(k_{1}\right) a_{k_{1}}^{+} a_{-k_{1}}^{+} \ldots v_{k_{i}} a_{k_{i}}^{+} a_{-k_{i}}^{+} \ldots f\left(k_{n}\right) a_{k_{n}}^{+} a_{-k_{n}}^{+}|0\rangle= \\
=c_{\Lambda} \sum_{n=1}^{\infty} \frac{1}{n !} \sum_{i=1}^{n} \sum_{k_{1}} f\left(k_{1}\right) a_{k_{1}}^{+} a_{-k_{1}}^{+} \ldots \sum_{k_{i}} v_{k_{i}} a_{k_{i}}^{+} a_{-k_{i}}^{+} \ldots \\
\ldots \sum_{k_{n}} f\left(k_{n}\right) a_{k_{n}}^{+} a_{-k_{n}}^{+}|0\rangle
\end{gathered}
$$

Note that in (2.4) only the terms with $k_{1} \neq \ldots \neq k_{i} \neq \ldots \neq k_{n}$ are different from zero.

Consider the operator

$$
\mathcal{A}_{\Lambda}^{+}=c_{\Lambda} \sum_{k} v_{k} a_{k}^{+} a_{-k}^{+},
$$

where $c_{\Lambda}$ is defined according to (2.3). By direct calculation and by analogy with the operator $\nu \sum_{p} v_{p} a_{p}^{+} a_{-p}^{+}$one obtains

$$
\begin{gathered}
\mathcal{A}_{\Lambda}^{+} f=c_{\Lambda} \sum_{n=0}^{\infty} \frac{1}{n !} \sum_{k} v_{k} a_{k}^{+} a_{-k}^{+} \sum_{k_{1}} f_{1}\left(k_{1}\right) a_{k_{1}}^{+} a_{-k_{1}}^{+} \ldots \sum_{k_{n}} f_{1}\left(k_{n}\right) a_{k_{n}}^{+} a_{-k_{n}}^{+}|0\rangle= \\
=c_{\Lambda} \sum_{n=0}^{\infty} \frac{1}{(n+1) !} \sum_{i=1}^{n+1} \sum_{k_{1}} f_{1}\left(k_{1}\right) a_{k_{1}}^{+} a_{-k_{1}}^{+} \ldots \sum_{k_{i}} v_{k_{i}} a_{k_{i}}^{+} a_{-k_{i}}^{+} \ldots \\
\ldots \sum_{k_{n+1}} f\left(k_{n+1}\right) a_{k_{n+1}}^{+} a_{-k_{n+1}}^{+}|0\rangle= \\
=c_{\Lambda} \sum_{n=1}^{\infty} \frac{1}{(n) !} \sum_{i=1}^{n} \sum_{k_{1}} f_{1}\left(k_{1}\right) a_{k_{1}}^{+} a_{-k_{1}}^{+} \ldots \sum_{k_{i}} v_{k_{i}} a_{k_{i}}^{+} a_{-k_{i}}^{+} \ldots \\
\ldots \sum_{k_{n}} f_{1}\left(k_{n}\right) a_{k_{n}}^{+} a_{-k_{n}}^{+}|0\rangle .
\end{gathered}
$$

Comparing (2.4) with (2.6) one concludes that

$$
\mathcal{A}_{\Lambda}^{I} f=A_{\Lambda}^{+} f .
$$

on coherent states of pairs (2.1).

Taking into account the last equality and formulas (1.7), (1.8), one can represent the Hamiltonian $H_{\Lambda, \nu}$, on coherent states of pairs (2.1) as follows:

$$
H_{\Lambda, \nu} f=\left(\sum_{\bar{p}}\left(\frac{p^{2}}{2 m}-\mu\right) a_{\bar{p}}^{+} a_{-\bar{p}}^{+}+c_{\Lambda} \sum_{p} v_{p} a_{p}^{+} a_{-p}^{+}+\right.
$$

ISSN 1027-3190.Укр. мат. журн., 2008, т. 60, № 9 


$$
\begin{gathered}
\left.+\nu \sum_{p} v_{p} a_{p}^{+} a_{-p}^{+}++\nu \sum_{p} v_{p} a_{-p} a_{p}+\mathcal{B}\right) f= \\
=\left(\sum_{\bar{p}}\left(\frac{p^{2}}{2 m}-\mu\right) a_{\bar{p}}^{+} a_{-\bar{p}}+c_{\Lambda} \sum_{p} v_{p} a_{p}^{+} a_{-p}^{+}+c_{\Lambda} \sum_{p} v_{p} a_{-p} a_{p}+\right. \\
\left.+\nu \sum_{p} v_{p} a_{p}^{+} a_{-p}^{+}+\nu \sum_{p} v_{p} a_{-p} a_{p}\right) f+\mathcal{B} f-c_{\Lambda} \sum_{p} v_{p} a_{-p} a_{p} f= \\
=H_{\Lambda, \nu}^{a} f+\mathcal{B} f-c_{\Lambda} \sum_{p} v_{p} a_{-p} a_{p} f,
\end{gathered}
$$

where

$$
H_{\Lambda, \nu}^{a}=\sum_{\bar{p}}\left(\frac{p^{2}}{2 m}-\mu\right) a_{\bar{p}}^{+} a_{\bar{p}}+\left(c_{\Lambda}+\nu\right) \sum_{p} v_{p} a_{p}^{+} a_{-p}^{+}+\left(c_{\Lambda}+\nu\right) \sum_{p} v_{p} a_{-p} a_{p}
$$

is the approximating Hamiltonian introduced by Bogolyubov. (In formula (2.8) we added and subtracted the operator $c_{\Lambda} \sum_{p} v_{p} a_{-p} a_{p}$.) Note that the constant $c_{\Lambda}$ (2.3) is defined by the function $f_{1}(p)$ and varies with $f(p)$.

\subsection{Eigenvalue and eigenvector problem for the approximating Hamiltonian}

$\boldsymbol{H}_{\Lambda, \nu}^{a}$. It is well known that the operator $H_{\Lambda, \nu}^{a}$ can be diagonal. Namely introduce the following operators:

$$
\begin{gathered}
\alpha_{k}=u_{k} a_{k}+w_{k} a_{-k}^{+}, \quad \alpha_{k}^{+}=u_{k} a_{k}^{+}+w_{k} a_{-k}, \\
\alpha_{-k}=u_{k} a_{-k}-w_{k} a_{k}^{+}, \quad \alpha_{-k}^{+}=u_{k} a_{-k}^{+}-w_{k} a_{k}, \\
u_{k}=(2)^{-\frac{1}{2}}\left[1+\varepsilon_{k}\left(\varepsilon_{k}^{2}+\left(c_{\Lambda}+\nu\right)^{2} v_{k}^{2}\right)^{-\frac{1}{2}}\right]^{\frac{1}{2}}, \\
w_{k}=(2)^{-\frac{1}{2}}\left[1-\varepsilon_{k}\left(\varepsilon_{k}^{2}+\left(c_{\Lambda}+\nu\right)^{2} v_{k}^{2}\right)^{-\frac{1}{2}}\right]^{\frac{1}{2}}, \\
\varepsilon_{k}=\frac{k^{2}}{2 m}-\mu, \quad E_{k}=\left(\varepsilon_{k}^{2}+\left(c_{\Lambda}+\nu\right)^{2} v_{k}^{2}\right)^{\frac{1}{2}} .
\end{gathered}
$$

Note that $u_{k}=1, w_{k}=0$ for $k \notin \mathcal{D}$ and we consider transformation (2.10) only for $k \in \mathcal{D}$.

The approximating Hamiltonian $H_{\Lambda, \nu}^{a}$ (2.9) can be represented through the operators $\alpha_{k}, \alpha_{k}^{+}, \alpha_{-k}, \alpha_{-k}^{+}$in the following diagonal form:

$$
H_{\Lambda, \nu}^{a}=\sum_{\bar{p}} E_{p} \alpha_{\bar{p}}^{+} \alpha_{\bar{p}}+\sum_{p}\left[\varepsilon_{p}-\left(\varepsilon_{p}^{2}+\left(c_{\Lambda}+\nu\right)^{2} v_{p}^{2}\right)^{\frac{1}{2}}\right],
$$

for $p \notin \mathcal{D}$, 


$$
H_{\Lambda, \nu}^{a}=\sum_{\bar{p}}\left(\frac{p^{2}}{2 m}-\mu\right) a_{\bar{p}}^{+} a_{\bar{p}}
$$

Denote by $\Phi_{0}^{a}$ the following state:

$$
\begin{gathered}
\Phi_{0}^{a}=\prod_{k}\left(1+f^{a}(k) a_{k}^{+} a_{-k}^{+}\right)|0\rangle, \\
f^{a}(k)=\frac{-w_{k}}{u_{k}} .
\end{gathered}
$$

It is easy to check by direct calculation that the state $\Phi_{0}^{a}$ is the vacuum state for the operators $\alpha_{k}, \alpha_{k}^{+}, \alpha_{-k}, \alpha_{-k}^{+}$. Indeed,

$$
\alpha_{p} \Phi_{0}^{a}=0, \quad \alpha_{-p} \Phi_{0}^{a}=0,
$$

because

$$
\begin{gathered}
\alpha_{p} \Phi_{0}^{a}=\left(w_{p}-w_{p}\right) \alpha_{-p}^{+} \prod_{k \neq p}\left(1+f^{a}(k) a_{k}^{+} a_{-k}^{+}\right)|0\rangle=0, \\
\alpha_{-p} \Phi_{0}^{a}=\left(-w_{p}+w_{p}\right) \alpha_{p}^{+} \prod_{k \neq p}\left(1+f^{a}(k) a_{k}^{+} a_{-k}^{+}\right)|0\rangle=0 .
\end{gathered}
$$

Therefore

$$
H_{\Lambda, \nu}^{a} \Phi_{0}^{a}=\sum_{P \subset \mathcal{D}}\left[\varepsilon_{p}-\left(\varepsilon_{p}^{2}+\left(c_{\Lambda}+\nu\right)^{2} v_{p}^{2}\right)^{\frac{1}{2}}\right] \Phi_{0}^{a},
$$

and this means that the vacuum state $\Phi_{0}^{a}$ is the eigenvector of $H_{\Lambda, \nu}^{a}$ with the eigenvalue $\sum_{P \subset \mathcal{D}}\left[\varepsilon_{p}-\left(\varepsilon_{p}^{2}+\left(c_{\Lambda}+\nu\right)^{2} v_{p}^{2}\right)^{\frac{1}{2}}\right]$.

It follows from (2.10) that $u_{k}>0$ because $v_{k} \neq 0,\left|\varepsilon_{k}-\left(\varepsilon_{k}^{2}+\left(c_{\Lambda}+\nu\right)^{2} v_{k}^{2}\right)^{-\frac{1}{2}}\right|<1$ in $\mathcal{D}$ and, thus, the function $f^{a}(k)$ is uniformly bounded in $\mathcal{D}$, i.e., $\left|f^{a}(k)\right|<f$. The state $\Phi_{0}^{a}(2.12)$ can be represented as follows:

$$
\begin{gathered}
\Phi_{0}^{a}=\sum_{n=0}^{\infty} \sum_{k_{1} \neq \ldots \neq k_{n}}^{\prime} f^{a}\left(k_{1}\right) \ldots f^{a}\left(k_{n}\right) a_{k_{1}}^{+} a_{-k_{1}}^{+} \ldots a_{k_{n}}^{+} a_{-k_{n}}^{+}|0\rangle= \\
=\sum_{n=0}^{\infty} \frac{1}{n !} \sum_{k_{1} \neq \ldots \neq k_{n}} f^{a}\left(k_{1}\right) \ldots f^{a}\left(k_{n}\right) a_{k_{1}}^{+} a_{-k_{1}}^{+} \ldots a_{k_{n}}^{+} a_{-k_{n}}^{+}|0\rangle \equiv \\
=\sum_{n=0}^{\infty} \frac{1}{n !} \sum_{k_{1}, \ldots, k_{n}} f^{a}\left(k_{1}\right) \ldots f^{a}\left(k_{n}\right) a_{k_{1}}^{+} a_{-k_{1}}^{+} \ldots a_{k_{n}}^{+} a_{-k_{n}}^{+}|0\rangle .
\end{gathered}
$$

In the last expression we added terms equal to zero with equal momenta. It has been proved in our paper [3] that on the state (2.12) with $\left|f^{a}(k)\right|<f$ the operator $\mathcal{B}$ can be estimates as follows:

$$
\left|\left(\Phi_{0}^{a}, \mathcal{B} \Phi_{0}^{a}\right)_{V}^{\prime}\right|<\frac{|g| v^{2}}{V} \alpha^{2} f^{4} e^{\alpha} f^{2}
$$

ISSN 1027-3190.Укр. мат. журн., 2008, т. 60, № 9 


$$
\begin{aligned}
\left|\left(\mathcal{B} \Phi_{0}^{a}, \mathcal{B} \Phi_{0}^{a}\right)_{V}^{\prime}\right| & \leq \frac{g v^{4}}{V^{2}}\left(6 \alpha^{4} f^{8} e^{\alpha} f^{2}+2 \alpha^{2} f^{4}+\alpha^{3} f^{6}\right), \\
\alpha & =\frac{8 \pi}{3(2 \pi)^{2}}[2 m(\mu+w)]^{\frac{3}{2}} .
\end{aligned}
$$

Then from (2.8), (2.15) one gets

$$
\begin{gathered}
\lim _{V \rightarrow \infty}\left|\left(\Phi_{0}^{a},\left[H_{\Lambda, \nu}-H_{\Lambda, \nu}^{a}-c_{\Lambda} \sum_{p} v_{p} a_{-p} a_{p}\right] \Phi_{0}^{a}\right)_{V}^{\prime}\right|=\lim _{V \rightarrow \infty}\left|\left(\Phi_{0}^{a}, \mathcal{B} \Phi_{0}^{a}\right)_{V}^{\prime}\right|=0 \\
\lim _{V \rightarrow \infty} \mid\left(\left[H_{\Lambda, \nu}-H_{\Lambda, \nu}^{a}-c_{\Lambda} \sum_{p} v_{p} a_{-p} a_{p}\right] \Phi_{0}^{a},\left[H_{\Lambda, \nu}-H_{\Lambda, \nu}^{a}-\right.\right. \\
\left.\left.-c_{\Lambda} \sum_{p} v_{p} a_{-p} a_{p}\right] \Phi_{0}^{a}\right)_{V}^{\prime} \mid=\lim _{V \rightarrow \infty}\left(\mathcal{B} \Phi_{0}^{a}, \mathcal{B} \Phi_{0}^{a}\right)_{V}^{\prime}=0 \\
\lim _{V \rightarrow \infty}\left\|\left[H_{\Lambda, \nu}-H_{\Lambda, \nu}^{a}-c_{\Lambda} \sum_{p} v_{p} a_{-p} a_{p}\right] \Phi_{0}^{a}\right\|_{V}^{\prime}=0 .
\end{gathered}
$$

Thus we note that inequalities (2.16), (2.17) are true not only for coherent states of pairs, but for arbitrary $f \in \mathcal{H}^{P}$ with wave functions of $n$ pairs $f_{n}\left(k_{1}, \ldots, k_{n}\right)$ satisfying conditions $\sup _{(k)_{n}}\left|f_{n}\left(k_{1}, \ldots, k_{n}\right)\right|<f^{n}, f<\infty$, see [3] formulas (5.8), (5.11). We already know that the state $\Phi_{0}^{a}$ is the eigenvector of the operator $H_{\Lambda, \nu}^{a}$ with the eigenvalue $\sum_{P \subset \mathcal{D}}\left[\varepsilon_{p}-\left(\varepsilon_{p}^{2}+\left(c_{\Lambda}+\nu\right)^{2} v_{p}^{2}\right)\right](\operatorname{see}(2.14))$.

Now consider the operator $c \sum_{p} v_{p} a_{-p} a_{p}$ on the state $\Phi_{0}^{a}$. One has

$$
\begin{gathered}
c_{\Lambda} \sum_{p} v_{p} a_{-p} a_{p} \Phi_{0}^{a}= \\
=c_{\Lambda} \sum_{n=0}^{\infty} \frac{1}{n !} \sum_{k_{1} \neq \ldots \neq k_{n}} \sum_{p \neq(k)_{n}} v_{p} f^{a}(p) f^{a}\left(k_{1}\right) \ldots f^{a}\left(k_{n}\right) a_{k_{1}}^{+} a_{-k_{1}}^{+} \ldots a_{k_{n}}^{+} a_{-k_{n}}^{+}|0\rangle .
\end{gathered}
$$

Further one has

$$
\begin{gathered}
\lim _{V \rightarrow \infty} \frac{1}{V}\left(c_{\Lambda} \sum_{p} v_{p} a_{-p} a_{p} \Phi_{0}^{a}, \Phi_{0}^{a}\right)_{V}^{\prime}= \\
=\lim _{V \rightarrow \infty} \frac{1}{V} c_{\Lambda} \sum_{p \neq(k)_{n}} v_{p} f^{a}(p) \sum_{n=0}^{\infty} \frac{1}{n !} \frac{1}{V^{n}} \sum_{k_{1} \neq \ldots \neq k_{n}}\left|f^{a}\left(k_{1}\right)\right|^{2} \ldots\left|f^{a}\left(k_{n}\right)\right|^{2}= \\
=g^{-1} c^{2} \sum_{n=0} \frac{1}{n !}\left(\int\left|f^{a}(k)\right|^{2} d k\right)^{n}=g^{-1} c^{2} e^{\int\left|f^{a}(k)\right|^{2} d k}
\end{gathered}
$$


because

$$
\begin{gathered}
\lim _{V \rightarrow \infty} \frac{1}{V} \sum_{p \neq(k)_{n}} v_{p} f^{a}(p)=g^{-1} c, \\
\lim _{V \rightarrow \infty} \frac{1}{V^{n}} \sum_{k_{1} \neq \ldots \neq k_{n}}\left|f^{a}\left(k_{1}\right)\right|^{2} \ldots\left|f^{a}\left(k_{n}\right)\right|^{2}=\left(\int\left|f^{a}(k)\right|^{2} d k\right)^{n}, \\
n \geq 1, \quad \lim _{V \rightarrow \infty} c_{\Lambda}=c, \\
\lim _{V \rightarrow \infty}\left(\Phi_{0}^{a}, \Phi_{0}^{a}\right)_{V}^{\prime}=e^{\int\left|f^{a}(k)\right|^{2} d k} .
\end{gathered}
$$

We have taken into account that in the integral sums one can neglect the hyperplanes $p=k_{1}, \ldots, p=k_{n}$ in the first sum and the hyperplanes $k_{1}=k_{2}, \ldots, k_{n-1}=k_{n}$ in the second sums. (We have omitted some details of proof. It will be presented in more complicated situation, connected with excited states of $\Phi_{0}^{a}$, in Section 4.)

If $f$ is a state (2.1) with an arbitrary wave function $f(k)$ of one pair, then one has

$$
\begin{gathered}
\lim _{V \rightarrow \infty} \frac{1}{V}\left(c_{\Lambda} \sum_{p} v_{p} a_{-p} a_{p} \Phi_{0}^{a}, f\right)_{V}^{\prime}= \\
=\lim _{V \rightarrow \infty} \frac{1}{V} c_{\Lambda} \sum_{n=0}^{\infty} \frac{1}{n !} \frac{1}{V^{n}} \sum_{p \neq(k)_{n}} v_{p} f^{a}(p) \sum_{k_{1} \neq \ldots \neq k_{n}} f^{a}\left(k_{1}\right) f\left(k_{1}\right) \ldots f^{a}\left(k_{n}\right) f\left(k_{n}\right)= \\
=c^{2} e^{\int f^{a}(k) f(k) d k} .
\end{gathered}
$$

The last formula can be interpreted as a proof that $\lim _{V \rightarrow \infty} \frac{1}{V} c_{\Lambda} \sum_{p} v_{p} a_{-p} a_{p} \Phi_{0}^{a}=c^{2} \Phi_{0}^{a}$ in the weak sense on coherent states.

It is obvious that

$$
\lim _{V \rightarrow \infty} \frac{1}{V^{2}}\left(c_{\Lambda} \sum_{p} v_{p} a_{-p} a_{p} \Phi_{0}^{a}, c_{\Lambda} \sum_{p} v_{p} a_{-p} a_{p} \Phi_{0}^{a}\right)_{V}^{\prime}=c^{4} e^{\int\left|f^{a}(k)\right|^{2} d k} .
$$

Thus, in the above described sense, the operator $c_{\Lambda} \sum_{p} v_{p} a_{-p} a_{p}$ on the state $\Phi_{0}^{a}$ tends in the thermodynamic limit to the operator of multiplication by $g^{-1} c^{2} V$

$$
\lim _{V \rightarrow \infty} c_{\Lambda} \sum_{p} v_{p} a_{-p} a_{p} \Phi_{0}^{a}=c^{2} \bar{V} \Phi_{0}^{a}
$$

where $\bar{V}$ denotes the infinite volume of the entire three-dimensional Euclidean space.

The state $\Phi_{0}^{a}$ is the eigenvector of the operator $H_{\Lambda, \nu}^{a}$ with eigenvalue $\sum_{p \subset \mathcal{D}}\left[\varepsilon_{p}-\right.$ $\left.-\left(\varepsilon_{p}^{2}+\left(c_{\Lambda}+\nu\right)^{2} v_{p}^{2}\right)^{\frac{1}{2}}\right]$. If follows from (2.14) and (2.15) that the same state $\Phi_{0}^{a}$ is the eigenvector of the operator $H_{\Lambda, \nu}^{a}-c_{\Lambda} \sum_{p} v_{p} a_{-p} a_{p}$ in the thermodynamic limit with the eigenvalue 


$$
\begin{aligned}
& \lim _{V \rightarrow \infty}\left(\sum_{p \subset \mathcal{D}}\left[\varepsilon_{p}-\left(\varepsilon_{p}^{2}+\left(c_{\Lambda}+\nu\right)^{2} v_{p}^{2}\right)^{\frac{1}{2}}\right]-c_{\Lambda}^{2} V\right)= \\
& =\bar{V}\left[\int\left(\varepsilon(p)-\left(\varepsilon(p)^{2}+(c+\nu)^{2} v(p)^{2}\right)^{\frac{1}{2}}\right)-c^{2}\right] .
\end{aligned}
$$

3. BCS Hamiltonian for infinite volume (in the entire Euclidean space). 3.1. Operator $\mathcal{A}_{\Lambda}$ in the thermodynamic limit. Define the operator $\mathcal{A}_{\Lambda}$ for one pair (one puts $\nu=0$ in $\left.\mathcal{A}_{\nu}\right)$

$$
\left(\mathcal{A}_{\Lambda} f_{1}\right)\left(k_{1}\right)=\left(\frac{2 k_{1}^{2}}{2 m}-2 \mu\right) f_{1}(k)+\frac{g}{V} \sum_{p} v_{k_{1}} v_{p} f_{1}(p)
$$

and denote it by $H_{2, \Lambda}$

$$
\left(H_{2, \Lambda} f_{1}\right)\left(k_{1}\right)=\left(\frac{2 k_{1}^{2}}{2 m}-2 \mu\right) f_{1}(k)+\frac{g}{V} \sum_{p} v_{k_{1}} v_{p} f_{1}(p) .
$$

In our previous paper [6] we have completely investigated the eigenvalue and eigenvector problem for the operator $H_{2, \Lambda}$ with finite and infinite cube $\Lambda$.

Now we want to explain in what sense the operator $H_{2, \Lambda}$ converges to $H_{2, \mathbb{R}^{3}} \equiv H_{2}$. For this purpose, we restrict ourselves to a potential $v_{k}$ with the following compact support $\mathcal{D}\left(k\left|\frac{k^{2}}{2 m}-\mu\right|<\omega\right), \omega>0$. Consider a continuous function $f_{1}(k)$ with support $\mathcal{D}$ and defined for arbitrary $k \in \mathcal{D}$, but not only for quasidiscrete $k=\frac{2 \pi}{L}\left(n_{1}, n_{2}, n_{3}\right)$, $n_{i} \in \mathbb{Z}, i=1,2,3$. The norm of the function $f_{1}(k)$ with these quasidiscrete $k$ is defined as follows:

$$
\left\|f_{1}\right\|_{\Lambda}=\left\{\frac{1}{V} \sum_{k}\left|f_{1}(k)^{2}\right|\right\}^{\frac{1}{2}}, \quad V=\frac{L^{3}}{(2 \pi)^{3}} .
$$

The norm of the function $f_{1}(k)$ with continuous momenta $k$ is defined as follows:

$$
\|f\|_{\mathbb{R}^{3}}=\|f\|=\left\{\int\left|f_{1}(k)\right|^{2} d k\right\}^{\frac{1}{2}} .
$$

It is obvious that $\|f\|_{\Lambda}$ tends to $\|f\|$ as $V \rightarrow \infty(L \rightarrow \infty)$ because the expression $\frac{1}{V} \sum_{k}\left|f_{1}(k)\right|^{2}$ is the Riemann sum which converges to $\int\left|f_{1}(k)\right|^{2} d k$, i.e.,

$$
\lim _{V \rightarrow \infty} \frac{1}{V} \sum_{k}\left|f_{1}(k)\right|^{2}=\int\left|f_{1}(k)\right|^{2} d k=\left\|f_{1}\right\| .
$$

For each of terms in $\left(H_{2, \Lambda} f_{1}\right)(k)$ we have

$$
\begin{aligned}
& \lim _{V \rightarrow \infty} \frac{1}{V} \sum_{k}\left|\left(\frac{2 k^{2}}{2 m}-2 \mu\right) f_{1}(k)\right|^{2}=\int\left|\left(\frac{2 k^{2}}{2 m}-2 \mu\right) f_{1}(k)\right|^{2} d k, \\
& \lim _{V \rightarrow \infty} \frac{1}{V} \sum_{k}\left|\frac{1}{V} \sum_{p} v(k) v_{p} f_{1}(p)\right|^{2}=\int|v(k)|^{2} d k\left|\int v(p) f_{1}(p) d p\right|^{2} .
\end{aligned}
$$


It is obvious that all the integrals are convergent due to continuity of $f_{1}(k), v(k)$ and the fact that they have compact support $\mathcal{D}$. (We denote by $v(k)$ potential $v_{k}$ with continuous momenta $k$.)

Define the operator $H_{2}$ for infinite $\Lambda=\mathbb{R}^{3}$ as follows:

$$
\left(H_{2} f_{1}\right)\left(k_{1}\right)\left(\frac{2 k_{1}^{2}}{2 m}-2 \mu\right) f_{1}\left(k_{1}\right)+v\left(k_{1}\right) \int v(p) f_{1}(p) d p .
$$

From the above obtained equalities (3.2) - (3.5) one concludes that

$$
\lim _{V \rightarrow \infty}\left\|H_{2, \Lambda} f_{1}\right\|_{\Lambda}=\left\|H_{2} f_{1}\right\|
$$

and in this sense the operator $H_{2, \Lambda}$ converges to the operator $H_{2}$ as $V \rightarrow \infty\left(\Lambda \rightarrow \mathbb{R}^{3}\right)$.

The operator $\mathcal{A}_{\Lambda}=\left.\mathcal{A}_{\Lambda, \nu}\right|_{\nu=0}$ is represented through the operators $H_{2, \Lambda}$ on wave functions of $n$ pairs as follows:

$$
\begin{gathered}
\left(\mathcal{A}_{\Lambda} f_{n}\right)\left(k_{1}, \ldots, k_{n}\right)= \\
=\left(\left[H_{2, \Lambda} \otimes I \ldots \otimes I+\ldots+I \otimes I \ldots \otimes H_{2, \Lambda}\right] f_{n}\right)\left(k_{1}, \ldots, k_{n}\right), \quad n \geq 1,
\end{gathered}
$$

where $I$ is the identity operator.

Define the following norm for finite and infinite $\Lambda$

$$
\begin{gathered}
\left\|f_{n}\right\|_{\Lambda}=\left\{\frac{1}{V^{n}} \sum_{k_{1}, \ldots, k_{n}}\left|f_{n}\left(k_{1}, \ldots, k_{n}\right)\right|^{2}\right\}^{\frac{1}{2}}, \\
\left\|f_{n}\right\|_{\Lambda=\mathbb{R}^{3}}=\left\|f_{n}\right\|=\left\{\int\left|f_{n}\left(k_{1}, \ldots, k_{n}\right)\right|^{2} d k_{1} \ldots d k_{n}\right\}^{\frac{1}{2}}
\end{gathered}
$$

for continuous functions with compact support $\mathcal{D}$ with respect to each of variables $k_{1}, \ldots, k_{n}$. It is obvious that

$$
\lim _{V \rightarrow \infty}\left\|f_{n}\right\|_{\Lambda}=\left\|f_{n}\right\|
$$

As a simple consequence one obtains that

$$
\lim _{V \rightarrow \infty}\left\|\mathcal{A}_{\Lambda} f_{n}\right\|_{\Lambda}=\left\|\mathcal{A} f_{n}\right\|
$$

if the operator $\mathcal{A}$ is defined as follows:

$$
\begin{gathered}
\left(\mathcal{A} f_{n}\right)\left(k_{1}, \ldots, k_{n}\right)= \\
=\left(\left[H_{2} \otimes I \ldots \otimes I+\ldots+I \otimes I \ldots \otimes H_{2}\right] f_{n}\right)\left(k_{1}, \ldots, k_{n}\right)= \\
=\sum_{i=1}^{n}\left[\left(\frac{2 k_{1}^{2}}{2 m}-2 \mu\right)+v\left(k_{i}\right) g \int v(p) f_{n}\left(k_{1}, \ldots, \frac{i}{p}, \ldots k_{n}\right) d p\right], \quad n \geq 1 .
\end{gathered}
$$

The BCS Hamiltonian $H_{\Lambda}=H_{\Lambda, \nu}=0$ on states of pairs $f(1.2)$ is represented according to (1.7), (1.8) as the sum of two operators $\mathcal{A}_{\Lambda}$ and $\mathcal{B}_{\Lambda}$

ISSN 1027-3190. Укр. мат. журн., 2008, m. 60, № 9 


$$
H_{\Lambda} f=\mathcal{A}_{\Lambda} f+\mathcal{B}_{\Lambda} f
$$

Taking into account estimates (2.16), (2.17) and formulas (3.9) - (3.11) one can conclude that for finite elements $f(1.2)$ (elements $f$ with $f_{n}\left(k_{1}, \ldots, k_{n}\right)=0$ for $n>n_{0}, n_{0}$ is finite number, and $\left.\left|f_{n}\left(k_{1}, \ldots, k_{n}\right)\right|<f^{n}, f<\infty\right)$

$$
\lim _{V \rightarrow \infty}\left\|H_{\Lambda} f\right\|_{V}^{\prime}=\lim _{V \rightarrow \infty}\left\|\mathcal{A}_{\Lambda} f\right\|_{V}^{\prime}=\|\mathcal{A} f\|
$$

Note that we need a finite element $f$ to have $\|A f\|<\infty$.

In this sense the BCS Hamiltonian is defined directly for infinite volume as follows:

$$
H f=\mathcal{A} f
$$

$$
(H f)_{n}\left(k_{1}, \ldots, k_{n}\right)=\left(H_{2} \otimes I \ldots \otimes I+\ldots+I \otimes I \ldots \otimes H_{2}\right) f_{n}\left(k_{1}, \ldots, k_{n}\right) .
$$

The last expression for the BCS Hamiltonian, directly for infinite volume, had been obtained in our paper [1] in 1970.

Remark 3.1. By a slight modification of formulas (3.8), (3.9) from our paper [1] one can obtain the following estimate for the operator $\mathcal{B}$ in the space $\mathcal{H}^{P}$ with norm $\|f\|_{V}(1.6)$

$$
\left\|\mathcal{B} f_{n}\right\|_{V} \leq g \frac{|V|\|V\|}{V^{\frac{1}{2}}} n(n-1)\|f\|_{V}
$$

From this estimate one can conclude that $\lim _{V \rightarrow \infty}\|\mathcal{B} f\|_{V}=0$ for finite $f \in \mathcal{H}^{P}$ and the estimate $\sup \left|f_{n}\left(k_{1}, \ldots, k_{n}\right)\right| \leq f^{n}, f<\infty$ is not imposed on $f_{n}\left(k_{1}, \ldots, k_{n}\right)$. We have formula

$$
(k)_{n}
$$

$$
\lim _{V \rightarrow \infty}\left\|H_{\Lambda} f\right\|=\|\mathcal{A} f\|=\|H f\| .
$$

Formulas (3.13)-(3.16) show in what sense $\lim _{V \rightarrow \infty} H_{\Lambda}=H$.

3.2. BCS and approximating Hamiltonians for infinite volume $[1,2]$. Now we show to prove that the BCS Hamiltonian for infinite volume represented by the operator $\mathcal{A}$ (3.14) coincides with the approximating Hamiltonian on coherent states of pairs. It can be done in full analogy with calculation performed in the Section 1.

Denote by $\mathcal{A}^{I}$ the part of operator $\mathcal{A}$ that describes the interaction of particles with opposite momenta and spin. It is defined as follows:

$$
\left(\mathcal{A}^{I} f\right)_{n}\left(k_{1}, \ldots, k_{n}\right)=\sum_{i=1}^{n} v\left(k_{i}\right) g \int v(p) f_{n}\left(k_{1}, \ldots, \frac{i}{p}, \ldots k_{n}\right) d p .
$$

The operator $\mathcal{A}^{I}$ coincides with the operator $\mathcal{A}^{+}=c \int v(k) a^{+}(k) a(-k) d k$ on coherent states of pairs

$$
f=\sum_{n=0}^{\infty} \frac{1}{n !} \int f\left(k_{1}\right) a^{+}\left(k_{1}\right) a^{+}\left(-k_{1}\right) d k_{1} \ldots \int f\left(k_{n}\right) a^{+}\left(k_{n}\right) a^{+}\left(-k_{n}\right) d k_{n}|0\rangle
$$


with $c=g \int v(p) f(p) d p$. (For the creation and annihilation operators with continuous momenta $k$ we use the notation $a^{+}(k), a(k)$.) Indeed,

$$
\begin{gathered}
\mathcal{A}^{I} f=c \sum_{n=1}^{\infty} \frac{1}{n !} \sum_{i=1}^{n} \int f\left(k_{1}\right) a^{+}\left(k_{1}\right) a^{+}\left(-k_{1}\right) d k_{1} \ldots \\
\ldots \int v\left(k_{i}\right) a^{+}\left(k_{i}\right) a^{+}\left(-k_{i}\right) d k_{i} \ldots \int f\left(k_{n}\right) a^{+}\left(k_{n}\right) a^{+}\left(-k_{n}\right) d k_{n}, \\
\mathcal{A}^{+} f=c \sum_{n=1}^{\infty} \frac{\mathcal{A}}{n !} \sum_{i=1}^{n} \int f\left(k_{1}\right) a^{+}\left(k_{1}\right) a^{+}\left(-k_{1}\right) d k_{1} \ldots \\
\ldots \int v\left(k_{i}\right) a^{+}\left(k_{i}\right) a^{+}\left(-k_{i}\right) d k_{i} \ldots \int f\left(k_{n}\right) a^{+}\left(k_{n}\right) a^{+}\left(-k_{n}\right) d k_{n}
\end{gathered}
$$

and thus

$$
\mathcal{A}^{I} f=\mathcal{A}^{+} f
$$

This means that the BCS Hamiltonian $H=\sum_{n=0}^{\infty} \otimes \sum_{i=1}^{n} I \otimes \ldots \otimes \frac{i}{H_{2}} \otimes \ldots \otimes I$ on the coherent states $f=e^{\int f(k) a^{+}(k) a^{+}(-k) d k}$ coincides with the operator

$$
H=\int\left(\frac{k^{2}}{2 m}-\mu\right) a^{+}(\bar{k}) a(\bar{k}) d k+c \int v(k) a^{+}(k) a^{+}(-k) d k .
$$

Now introduce the approximating Hamiltonian

$$
\begin{aligned}
& H^{\text {appr }}=H+c \int v(k) a(-k) a(k) d k-c \int v(k) a(-k) a(k) d k= \\
& =\left\{\int\left(\frac{k^{2}}{2 m}-\mu\right) a^{+}(\bar{k}) a(\bar{k}) d k+c \int v(k) a^{+}(k) a^{+}(-k) d k+\right. \\
& \left.\quad+c \int v(k) a(-k) a(k) d k\right\}-c \int v(k) a(-k) a(k) d k .
\end{aligned}
$$

Note that the operator $c \int v(k) a(-k) a(k) d k$ on the coherent states of pairs $f(3.17)$ is defined as follows:

$$
\begin{gathered}
c \int v(k) a(-k) a(k) d k f=g^{-1} c^{2} \bar{V} f, \\
\frac{1}{V} c \int v(k) a(-k) a(k) d k f=c^{2} f .
\end{gathered}
$$

We use the formula $\delta(0)=\bar{V}$.

Note that the constant $c=g \int v(k) f(k) d k$ is the same for all $n$, because in integral $\int v(p) f(p) d p$ one can neglect behavior of the integrand $v(k) f(k)$ in the points ISSN 1027-3190. Укр. мат. журн., 2008, m. 60, № 9 
$p=k_{1}, \ldots, k_{n}$. According to the Fermi statistics, the operator $c \int v(p) a(-p) a(p) d p$ annihilates only two operators $a^{+}(p) a^{+}(-p)$ from one pair with momenta $p$ and $(-p)$.

Finally, on coherent states $f$ one obtains

$$
\begin{gathered}
H^{\mathrm{appr}} f=\left(\int\left(\frac{k^{2}}{2 m}-\mu\right) a^{+}(\bar{k}) a(\bar{k}) d k+c \int v(k) a^{+}(k) a^{+}(-k) d k+\right. \\
\left.+c \int v(k) a(-k) a(k) d k-c^{2} g^{-1} \bar{V} I\right)
\end{gathered}
$$

where $I$ is the identity operator.

In this sense, for infinite volume, the BCS Hamiltonian $H$ coincides with the approximating Hamiltonian $H^{\text {appr }}$ on coherent states.

The coherent state $\Phi_{0}^{a}=e^{\int f_{0}^{a}(k) a^{+}(k) a^{+}(-k) d k}|0\rangle$ is the eigenvector of the approximating Hamiltonian as well as of the BCS Hamiltonian with the following eigenvalue: $\bar{V} \int\left[\varepsilon(p)-\left(\varepsilon(p)^{2}+c^{2}+v(p)^{2}\right)\right] d p, c=\int v(p) f_{0}^{a}(p) d p$.

4. Hamiltonian $\boldsymbol{H}_{\Lambda, \nu}$ on excited states. 4.1. Excited states. Denote by $f_{(p)_{l},(g)_{m}}=$ $=f_{p_{1}, \ldots, p_{l} ; q_{1}, \ldots, q_{m}}$ the following state:

$$
\begin{gathered}
f_{(p)_{l},(q)_{m}}=a_{\bar{p}_{1}}^{+} \ldots a_{\bar{p}_{l}}^{+} a_{q_{1}}^{+} a_{-q_{1}}^{+} \ldots a_{q_{m}}^{+} a_{-q_{m}}^{+} f= \\
=a_{\bar{p}_{1}}^{+} \ldots a_{\bar{p}_{l}}^{+} a_{q_{1}}^{+} a_{-q_{1}}^{+} \ldots a_{q_{m}}^{+} a_{-q_{m}}^{+} \sum_{n=0}^{\infty} \frac{1}{n !} \times \\
\times \sum_{k_{1} \neq \ldots \neq k_{n}} f_{n}\left(k_{1}, \ldots, k_{n}\right) a_{k_{1}}^{+} a_{-k_{1}}^{+} \ldots a_{k_{n}}^{+} a_{-k_{n}}^{+}|0\rangle
\end{gathered}
$$

where $f$ was defined by (1.2) and $f \in \mathcal{H}_{V}^{P}$. We say that $f_{(p)_{l},(q)_{m}}$ is an excited state with momenta $p_{1}, \ldots, p_{l}$ of $l$ particles and momenta $\left(q_{1},-q_{1}\right), \ldots,\left(q_{m},-q_{m}\right)$ of $m$ pairs. We assume that any two momenta $\left(p_{i}, p_{j}\right)$ do not coincide with some pairs of momenta from the set $\left(k_{1},-k_{1}, \ldots, k_{n},-k_{n}\right)=(k)_{n}, n=1,2, \ldots, k_{i} \subset \mathcal{D}$ or from the set $\left(q_{1},-q_{1}, \ldots, q_{m},-q_{m}\right)=(q)_{m}, q_{j} \subset \mathcal{D}$, but some $p_{i}$ can coincide with some momenta from the sets $(k)_{n}$. If some momenta from $(p)_{l}$ coincide with some momenta from $(q)_{m}$, then $f_{(p)_{l},(q)_{m}}=0$.

We consider $f_{(p)_{l},(q)_{m}}$ as an element of $\mathcal{H}^{F}$ with respect to momenta $(p)_{l},(q)_{m}$ and as an element of $\mathcal{H}_{V}^{P}$ with respect to momenta $(k)_{n}, n=1,2, \ldots$, i.e., $f_{(p)_{l},(q)_{m}} \in$ $\in \mathcal{H}^{F} \otimes \mathcal{H}_{V}^{P}$. We will use the notation $\left(f_{(p)_{l},(q)_{m}}, g_{(p)_{l},(q)_{m}}\right)_{V}^{\prime}$ for the scalar product of two elements $f_{(p)_{l},(q)_{m}}$ and $g_{(p)_{l},(q)_{m}}$ from $\mathcal{H}^{F} \otimes \mathcal{H}_{V}^{P}$.

The scalar product of two sequences $f_{(p)_{l},(q)_{m}}$ and $g_{(p)_{l},(q)_{m}}$ is equal to the following expression:

$$
\begin{gathered}
\left(f_{(p)_{l},(q)_{m}}, g_{(p)_{l},(q)_{m}}\right)_{V}^{\prime}= \\
=\sum_{n=0}^{\infty} \frac{1}{V^{n}} \sum_{(k)_{n} \neq(p)_{l} \neq(q)_{m}}^{\prime} \frac{}{f_{n}\left(k_{1}, \ldots, k_{n}\right)} g_{n}\left(k_{1}, \ldots, k_{n}\right)=
\end{gathered}
$$




$$
=\sum_{n=0}^{\infty} \frac{1}{n !} \frac{1}{V^{n}} \sum_{(k)_{n} \neq(p)_{l} \neq(q)_{m}}^{\prime} \overline{f_{n}\left(k_{1}, \ldots, k_{n}\right)} g_{n}\left(k_{1}, \ldots, k_{n}\right) .
$$

Note that $\sum_{(k)_{n} \neq(p)_{l} \neq(q)_{m}}^{\prime}$ means that one considers only $k_{1} \neq k_{2} \neq \ldots \neq k_{n}$ and identifies $(k)_{n}$ that differs only by permutation. Consider the action of $H_{\Lambda, \nu}$ on $f_{(p)_{l},(q)_{m}}$ and for the sake of simplicity we consider only operators $a_{p}^{+}$with spins $H$. By analogy with (1.3) one obtains

$$
\begin{aligned}
& H_{\Lambda, \nu} f_{(p)_{l},(q)_{m}}= \\
& =\sum_{n=0}^{\infty} \frac{1}{n !} \sum_{k_{1}, \ldots, k_{n}}\left\{\left[\sum_{i=1}^{l}\left(\frac{p_{i}^{2}}{2 m}-\mu\right)+\sum_{i=1}^{m}\left(\frac{2 q_{j}^{2}}{2 m}-2 \mu\right)+\sum_{i=1}^{n}\left(\frac{2 k_{i}^{2}}{2 m}-2 \mu\right)\right] \times\right. \\
& \times f_{n}\left(k_{1}, \ldots, k_{n}\right)+\frac{g}{V} \sum_{i=1}^{n}\left[\sum_{p} v_{k_{i}} v_{p} f_{n}\left(k_{1}, \ldots, \frac{1}{p}, \ldots, k_{n}\right)-\right. \\
& -\sum_{1=j \neq i}^{n} v_{k_{i}} v_{k_{j}} f_{n}\left(k_{1}, \ldots, \frac{i}{k_{j}}, \ldots, k_{n}\right)-\sum_{j=1}^{l} v_{k_{i}} v_{p_{j}} f_{n}\left(k_{1}, \ldots, \frac{i}{p_{j}}, \ldots, k_{n}\right)- \\
& \left.\left.-\sum_{j=1}^{m} v_{k_{i}} v_{q_{j}} f_{n}\left(k_{1}, \ldots, \frac{i}{q_{j}}, \ldots, k_{n}\right)\right]\right\} \times \\
& \times a_{p_{1}}^{+} \ldots a_{p_{l}}^{+} a_{q_{1}}^{+} a_{-q_{1}}^{+} \ldots a_{q_{m}}^{+} a_{-q_{m}}^{+} a_{k_{1}}^{+} a_{-k_{1}}^{+} \ldots a_{k_{n}}^{+} a_{-k_{n}}^{+}|0\rangle+ \\
& +\frac{g}{V} \sum_{j=1}^{m} \sum_{k_{n+1}} v_{k_{n+1}} v_{q_{j}} f_{n}\left(k_{1}, \ldots, k_{n}\right) \times \\
& \times a_{p_{1}}^{+} \ldots a_{p_{l}}^{+} a_{q_{1}}^{+} a_{-q_{1}}^{+} \ldots \stackrel{j}{V}^{j} \ldots a_{q_{m}}^{+} a_{-q_{m}}^{+} a_{k_{1}}^{+} a_{-k_{1}}^{+} \ldots a_{k_{n}}^{+} a_{-k_{n}}^{+} a_{k_{n+1}}^{+} a_{-k_{n+1}}^{+}|0\rangle+ \\
& +\nu \sum_{i=1}^{n} v_{k_{i}} f_{n-1}\left(k_{1}, \ldots, \vee^{i} \ldots, k_{n}\right) a_{p_{1}}^{+} \ldots a_{p_{l}}^{+} a_{q_{1}}^{+} a_{-q_{1}}^{+} \ldots \\
& \ldots a_{q_{m}}^{+} a_{-q_{m}}^{+} a_{k_{1}}^{+} a_{-k_{1}}^{+} \ldots a_{k_{n}}^{+} a_{-k_{n}}^{+}|0\rangle+ \\
& +\nu \sum_{p \neq(k)_{n} \neq(p)_{l} \neq(q)_{m}} v_{p} f_{n+1}\left(p, k_{1}, \ldots, k_{n}\right) a_{p_{1}}^{+} \ldots a_{p_{l}}^{+} a_{q_{1}}^{+} a_{-q_{1}}^{+} \ldots \\
& \ldots a_{q_{m}}^{+} a_{-q_{m}}^{+} a_{k_{1}}^{+} a_{-k_{1}}^{+} \ldots a_{k_{n}}^{+} a_{-k_{n}}^{+}|0\rangle+ \\
& +\nu \sum_{j=1}^{m} v_{q_{j}} f_{n}\left(k_{1}, \ldots, k_{n}\right) a_{p_{1}}^{+} \ldots a_{p_{l}}^{+} a_{q_{1}}^{+} a_{-q_{1}}^{+} \ldots \vee^{j} \ldots-q_{m}^{+} a_{k_{1}}^{+} a_{-k_{1}}^{+} \ldots a_{k_{n}}^{+} a_{-k_{n}}^{+}|0\rangle .
\end{aligned}
$$

ISSN 1027-3190. Укр. мат. журн., 2008, m. 60, № 9 
This yields

$$
\begin{gathered}
H_{\Lambda, \nu} f_{(p)_{l},(q)_{m}}= \\
=\left[\sum_{i=1}^{l}\left(\frac{p_{i}^{2}}{2 m}-\mu\right) I+\sum_{i=1}^{m}\left(\frac{2 q_{j}^{2}}{2 m}-2 \mu\right) I\right] f_{(p)_{l},(q)_{m}}+ \\
+\left(\mathcal{A}_{\Lambda}+\mathcal{B}_{(p)_{l},(q)_{m}}\right) f_{(p)_{l},(q)_{m}}+\mathcal{C}(p)_{l},(q)_{m} f_{(p)_{l},(q)_{m}}+ \\
+\left[\nu \sum_{p} v_{p} a_{p}^{+} a_{-p}^{+}+\nu \sum_{p} v_{p} a_{-p} a_{p}\right] f_{(p)_{l},(q)_{m}},
\end{gathered}
$$

where $\mathcal{A}_{\Lambda}$ is defined by the third and fourth term, the operator $\mathcal{B}_{(p)_{l},(q)_{m}}$ is defined by the fifth, sixth and seventh terms, the operator $\mathcal{C}_{(p)_{l},(q)_{m}}$ is defined by the eighth term, the operator $\nu \sum_{p} v_{p} a_{p}^{+} a_{-p}^{+}$is defined by the ninth term and the operator $\nu \sum_{p} v_{p} a_{-p} a_{p}$ is defined by the last two terms, $I$ is the identity operator.

4.2. Estimates for the operators $\mathcal{B}_{(p)_{l},(q)_{m}}, \mathcal{C}_{(p)_{l},(q)_{m}}$. We restrict ourselves to $f_{n}\left(k_{1}, \ldots, k_{n}\right)$ such that $\left|f_{n}\left(k_{1}, \ldots, k_{n}\right)\right| \leq f^{n}$. For the operator $\mathcal{B}_{(p)_{l},(q)_{m}}$ one has (see detail in [4])

$$
\begin{aligned}
& \left|\left(f_{(p)_{l},(q)_{m}}, \mathcal{B}_{(p)_{l},(q)_{m}} f_{(p)_{l},(q)_{m}}\right)_{V}^{\prime}\right| \leq \frac{|g| v^{2}}{V} \sum_{n=2}^{\infty} \frac{N^{n}}{V^{n}} \frac{n(n+l+m-1)}{n !} f^{2 n} \leq \\
& \leq \frac{|g| v^{2}(l+m+1)}{V} \sum_{n=2}^{\infty} \frac{\alpha^{2} f^{2 n}}{(n-2) !} \leq \frac{|g| v^{2}(l+m+1)}{V} \alpha^{2} f^{4} e^{\alpha} f^{2} \\
& \left(\left\|\mathcal{B}_{(p)_{l},(q)_{m}} f_{(p)_{l},(q)_{m}}\right\|_{V}^{\prime}\right)^{2}=\left(\mathcal{B}_{(p)_{l},(q)_{m}} f_{(p)_{l},(q)_{m}}, \mathcal{B}_{(p)_{l},(q)_{m}} f_{(p)_{l},(q)_{m}}\right) \leq \\
& \leq \frac{g^{2} v^{4}}{V^{2}} \sum_{n=2}^{\infty} \frac{N^{n}}{V^{n}} \frac{(n(n+l+m-1))^{2}}{n !} f^{2 n} \leq \frac{g^{2} v^{4}}{V^{2}} \sum_{n=2}^{\infty} \frac{\alpha^{n}(n(n+l+m-1))^{2}}{n !} f^{2 n}
\end{aligned}
$$

It is obvious that the last series is convergent.

For the operator $\mathcal{C}_{(p)_{l},(q)_{m}}$ one has

$$
\begin{gathered}
\left|\left(f_{(p)_{l},(q)_{m}}, \mathcal{C}_{(p)_{l},(q)_{m}} f_{(p)_{l},(q)_{m}}\right)_{V}^{\prime}\right| \leq \\
\leq \sum_{n=0}^{\infty} \frac{N^{n}}{V^{n}} \sum_{k_{1} \neq \ldots \neq k_{n}}\left|f_{n}\left(k_{1}, \ldots, k_{n}\right)\right|^{2} \frac{|g|}{V} \sum_{j=1}^{m}\left|V_{g_{j}, q_{j}}\right| \leq \\
\leq \sum_{n=0}^{\infty} \frac{N^{n}}{V^{n}} \frac{1}{n !} f^{2 n} \frac{v^{2}|g| m}{V} \frac{|g|}{V} m v^{2} e^{\alpha} f^{2}, \\
\left(\left\|\mathcal{C}_{(p)_{l},(q)_{m}} f_{(p)_{l},(q)_{m}}\right\|_{V}^{\prime}\right)^{2}=\left(\mathcal{C}_{(p)_{l},(q)_{m}} f_{(p)_{l},(q)_{m}}, \mathcal{C}_{(p)_{l},(q)_{m}} f_{(p)_{l},(q)_{m}}\right)_{V}^{\prime} \leq
\end{gathered}
$$




$$
\begin{gathered}
\leq \sum_{n=0}^{\infty} \frac{1}{V^{n}} \sum_{k_{1} \neq \ldots \neq k_{n}}^{\prime}\left(\frac{g^{2}}{V^{2}} \sum_{i=1}^{m} \sum_{k_{n+1}}\left|V_{k_{n+1}, q_{i}}\right|^{2}+\frac{g^{2}}{V^{2}} \sum_{i \neq j=1}^{m}\left|V_{q_{i}, q_{i}}\right|\left|V_{q_{j}, q_{j}}\right|\right) \times \\
\times\left|f_{n}\left(k_{1}, \ldots, k_{n}\right)\right|^{2} \leq \\
\leq \sum_{n=0}^{\infty} \frac{N^{n}}{V^{n}} f^{2 n}\left(\frac{g^{2}}{V^{2}} \alpha v^{4}+\frac{g^{2}}{V^{2}} m^{2} v^{4}\right) \leq \frac{g^{2} e^{\alpha} f^{2}}{V}\left(\alpha v^{4} m+\frac{m^{2} v^{4}}{V}\right) .
\end{gathered}
$$

Thus, the norms of the operators $\mathcal{B}_{(p)_{l},(q)_{m}}, \mathcal{C}_{(p)_{l},(q)_{m}}$ on elements $f_{(p)_{l},(q)_{m}}$ tend to zero as $V$ tends to infinity.

By analogy with Section 2 we have the following representation of the operator $H_{\Lambda, \nu}$ through the operator $H_{\Lambda, \nu}^{a}$ on the states $f_{(p)_{l},(q)_{m}}$

$$
\begin{gathered}
H_{\Lambda, \nu} f_{(p)_{l},(q)_{m}}= \\
=\left(\sum_{\bar{p}}\left(\frac{p^{2}}{2 m}-\mu\right) a_{\bar{p}}^{+} a_{\bar{p}}+c_{\Lambda} \sum_{p} v_{p} a_{p}^{+} a_{-p}^{+}+\nu \sum_{p} v_{p} a_{p}^{+} a_{-p}^{+}+\nu \sum_{p} v_{p} a_{-p} a_{p}+\right. \\
\left.+\mathcal{B}_{(p)_{l},(q)_{m}}+\mathcal{C}_{(p)_{l},(q)_{m}}\right) f_{(p)_{l},(q)_{m}}= \\
=\left(\sum_{\bar{p}}\left(\frac{p^{2}}{2 m}-\mu\right) a_{\bar{p}}^{+} a_{\bar{p}}+\left(c_{\Lambda}+\nu\right) \sum_{p} v_{p} a_{p}^{+} a_{-p}^{+}+\right. \\
\left.+\left(c_{\Lambda}+\nu\right) \sum_{p} v_{p} a_{-p} a_{p}+\mathcal{B}_{(p)_{l},(q)_{m}}+\mathcal{C}_{(p)_{l},(q)_{m}}-c_{\Lambda} \sum_{p} v_{p} a_{-p} a_{p}\right) f_{(p)_{l},(q)_{m}}= \\
=\left(H_{\Lambda, \nu}^{a}+\mathcal{B}_{(p)_{l},(q)_{m}}+\mathcal{C}_{(p)_{l},(q)_{m}}-c_{\Lambda} \sum_{p} v_{p} a_{-p} a_{p}\right) f_{(p)_{l},(q)_{m}} .
\end{gathered}
$$

From the above obtained estimates for the operators $\mathcal{B}_{(p)_{l},(q)_{m}}, \mathcal{C}_{(p)_{l},(q)_{m}}$ one can prove the following theorem.

Theorem 4.1. On the states of excited pairs $f_{(p)_{l},(q)_{m}}$, one has

$$
\begin{gathered}
\lim _{V \rightarrow \infty}\left\|\left(H_{\Lambda, \nu}-H_{\Lambda, \nu}^{a}-c_{\Lambda} \sum_{p} v_{p} a_{-p} a_{p}\right) f_{(p)_{l},(q)_{m}}\right\|_{V}^{\prime}= \\
=\lim _{V \rightarrow \infty}\left\|\left(\mathcal{B}_{(p)_{l},(q)_{m}}+\mathcal{C}_{(p)_{l},(q)_{m}}\right) f_{(p)_{l},(q)_{m}}\right\|_{V}^{\prime}=0 .
\end{gathered}
$$

It is well known that the states $f_{(p)_{l},(q)_{m}}$ with $f=\Phi_{0}^{a}$ and the operators $\alpha_{q_{1}}^{+} \alpha_{-q_{1}}^{+} \ldots \alpha_{q_{m}}^{+} \alpha_{-q_{m}}^{+}$instead of the operators $a_{q_{1}}^{+} a_{-q_{1}}^{+} \ldots a_{q_{m}}^{+} a_{-q_{m}}^{+}$, where 


$$
\begin{aligned}
\Phi_{0}^{a} & =\sum_{n=0}^{\infty} \frac{1}{n !} \sum_{k_{1} \neq \ldots \neq k_{n}} f_{0}^{a}\left(k_{1}\right) \ldots f_{0}^{a}\left(k_{n}\right) a_{k_{1}}^{+} a_{-k_{1}}^{+} \ldots a_{k_{n}}^{+} a_{-k_{n}}^{+}|0\rangle= \\
& =\sum_{n=0}^{\infty} \frac{1}{n !} \sum_{k_{1}, \ldots, k_{n}} f_{0}^{a}\left(k_{1}\right) \ldots f_{0}^{a}\left(k_{n}\right) a_{k_{1}}^{+} a_{-k_{1}}^{+} \ldots a_{k_{n}}^{+} a_{-k_{n}}^{+}|0\rangle,
\end{aligned}
$$

are the eigenvectors of the approximating Hamiltonian $H_{\Lambda, \nu}^{a}$ with eigenvalues

$$
\sum_{i=1}^{l} E_{p_{i}}+2 \sum_{i=1}^{m} E_{q_{i}}+\sum_{p}\left[\varepsilon_{p}-\sqrt{\varepsilon_{p}^{2}+(c+\nu)^{2} v_{p}^{2}}\right] .
$$

From the estimates established above one obtains the following theorem.

Theorem 4.2. If the states of pairs $f=\left(1,0, f_{1}\left(k_{1}\right), \ldots, f_{n}\left(k_{1}, \ldots, k_{n}\right), \ldots\right)$ satisfy the conditions

$$
\left|f_{n}\left(k_{1}, \ldots, k_{n}\right)\right| \leq f^{n}, \quad f<\infty, \quad n>1,
$$

uniformly with respect to $V$ and have supports in $\mathcal{D}^{n}$, then the expressions

$$
\begin{gathered}
\mid\left[H_{\Lambda}-\mathcal{A}_{\Lambda}-\sum_{i=1}^{l}\left(\frac{p_{i}^{2}}{2 m}-\mu\right) I-\sum_{i=1}^{m}\left(\frac{g_{i}^{2}}{2 m}-\mu\right) I-\right. \\
\left.\quad-\nu \sum_{p} v_{p} a_{p}^{+} a_{-p}^{+}-\nu \sum_{p} v_{p} a_{-p} a_{p}\right]\left.f_{(p)_{l},(q)_{m}}\right|_{V} ^{\prime}
\end{gathered}
$$

tend to zero as $V \rightarrow \infty$ for arbitrary fixed $l$ and $m$.

4.3. Approximating Hamiltonian on the states $f_{(p)_{l},(q)_{m}}$. In this subsection, we restrict ourselves to the state of pairs such that

$$
f=\left(1,0, f_{1}^{0}\left(k_{1}\right), f_{1}^{0}\left(k_{2}\right), \ldots, f_{1}^{0}\left(k_{n}\right), \ldots\right), \quad\left|f_{1}^{0}(k)\right| \leq f<\infty
$$

and define on these $f_{(p)_{l},(q)_{m}}$ the following approximating Hamiltonian:

$$
\begin{gathered}
H_{\Lambda, \nu}^{a}=\sum_{\bar{p}}\left(\frac{p^{2}}{2 m}-\mu\right) a_{\bar{p}}^{+} a_{\bar{p}}+\left(c_{\Lambda}+\nu\right) \sum_{p} v_{p} a_{p}^{+} a_{-p}+ \\
+\left(c_{\Lambda}+\nu\right) \sum_{p} v_{p} a_{-p} a_{p}-g^{-1} c_{\Lambda}^{2} V \\
c_{\Lambda}=\frac{g}{V} \sum_{p} v_{p} f_{1}^{0}(p) .
\end{gathered}
$$

By analogy with the calculations performed in Section 2, we have

$$
\begin{gathered}
\mathcal{A}^{I} f_{(p)_{l},(q)_{m}}=c_{\Lambda} \sum_{p} v_{p} a_{p}^{+} a_{-p}^{+} f_{(p)_{l},(q)_{m}}, \\
c_{\Lambda} \sum_{p} v_{p} a_{-p} a_{p} f_{(p)_{l},(q)_{m}}=c_{\Lambda} \sum_{n=1}^{\infty} \frac{1}{(n-1) !} \sum_{k_{1}} f_{1}^{0}\left(k_{1}\right) a_{k_{1}}^{+} a_{-k_{1}}^{+} \ldots
\end{gathered}
$$




$$
\begin{gathered}
\ldots \sum_{k_{n}-1} f_{1}^{0}\left(k_{n}-1\right) a_{k_{n}-1}^{+} a_{-k_{n}-1}^{+} \sum_{p \neq(k)_{n-1}, p \neq(p)_{l}, p \neq(g)_{m}} v_{p} f_{1}^{0}(p) a_{p_{1}}^{+} \ldots \\
\ldots a_{p_{l}}^{+} a_{q_{1}}^{+} a_{-q_{1}}^{+} \ldots a_{q_{m}}^{+} a_{-q_{m}}^{+}|0\rangle+c_{\Lambda} \sum_{i=1}^{m} v_{q_{i}} f_{(p)_{l},(\stackrel{i}{q})_{m}},
\end{gathered}
$$

where $(\stackrel{i}{q})_{m} \equiv\left(q_{1}, \ldots, \stackrel{i}{\vee}, \ldots, q_{m}\right)$.

4.4. Operator $c_{\Lambda} \sum_{p} v_{p} a_{-p} a_{p}$ on excited states. Now consider in detail the operator $c_{\Lambda} \sum_{p} v_{p} a_{-p} a_{p}$ on the states $f_{(p)_{l},(q)_{m}}$. One obtains

$$
\begin{gathered}
c_{\Lambda} \sum_{p} v_{p} a_{-p} a_{p} f_{(p)_{l},(q)_{m}}= \\
=g^{-1} c_{\Lambda}^{2} V f_{(p)_{l},(q)_{m}}+c_{\Lambda} \mathcal{B}_{(p)_{l},(q)_{m}}^{1} f_{(p)_{l},(q)_{m}}+c_{\Lambda} \sum_{i=1}^{m} v_{q_{i}} f_{(p)_{l},(\stackrel{)}{q})_{m}},
\end{gathered}
$$

where the operator $\mathcal{B}_{(p)_{l},(q)_{m}}^{1}$ is defined as follows:

$$
\begin{gathered}
\mathcal{B}_{(p)_{l},(q)_{m}}^{1} f_{(p)_{l},(q)_{m}}= \\
=-c_{\Lambda} a_{p_{1}}^{+} \ldots a_{p_{l}}^{+} a_{q_{1}}^{+} a_{-q_{1}}^{+} \ldots a_{q_{m}}^{+} a_{-q_{m}}^{+} \sum_{n=1}^{\infty} \frac{1}{n !} \sum_{k_{1}} f_{0}^{a}\left(k_{1}\right) a_{k_{1}}^{+} a_{-k_{1}}^{+} \ldots \\
\ldots \sum_{k_{n}} f_{0}^{a}\left(k_{n}\right) a_{k_{n}}^{+} a_{-k_{n}}^{+}|0\rangle v_{k=(k)_{n}, k=(p) l_{l}, k=(q)_{m} \mid k_{1} \neq k_{2} \neq k_{n}} v_{k} f_{0}^{a}(k) .
\end{gathered}
$$

One obtains the following estimates [4]:

$$
\begin{gathered}
\frac{1}{V}\left|\left(f_{(p)_{l},(q)_{m}}, \mathcal{B}_{(p)_{l},(q)_{m}}^{1} f_{(p)_{l},(q)_{m}}\right)_{V}^{\prime}\right| \leq \frac{1}{V} v(l+m+1) \alpha f^{3} e^{\alpha f^{2}}, \\
\frac{1}{V}\left(\mathcal{B}_{(p)_{l},(q)_{m}}^{1} f_{(p)_{l},(q)_{m}}, \mathcal{B}_{(p)_{l},(q)_{m}}^{1} f_{(p)_{l},(q)_{m}} f_{(p)_{l},(q)_{m}}\right)_{V}^{\prime} \leq \\
\leq \frac{1}{V} v^{2}(l+m+1)^{2}\left(\alpha f^{4}+2 \alpha^{2} f^{6} e^{\left.\alpha f^{2}\right)},\right. \\
\frac{1}{V}\left|\left(\sum_{i=1}^{m} v_{q_{i}} f_{(p)_{l},(\stackrel{i}{q})_{m}}, \sum_{i=1}^{m} v_{q_{i}} f_{(p)_{l},(q)_{m}}\right)_{V}^{\prime}\right| \leq \frac{1}{V} v^{2} m^{2} e^{\alpha f^{2}} .
\end{gathered}
$$

From these estimates one obtains the following theorem.

Theorem 4.3. The expressions

$$
\begin{gathered}
\frac{1}{V}\left(f_{(p)_{l},(q)_{m},\left(H_{\Lambda}\right.}-H_{\Lambda, \nu}^{a}-g^{-1} c_{\Lambda}^{2} V-c_{\Lambda} \mathcal{B}_{(p)_{l},(q)_{m}}^{1}\right) f_{(p)_{l},(q)_{m}}- \\
\left.-c_{\Lambda} \sum_{i=1}^{m} v_{q_{i}} f{ }_{(p)_{l},(\stackrel{i}{q})_{m}}^{\prime}\right)_{V}^{\prime} \\
\frac{1}{V} \|\left[\left(H_{\Lambda}-H_{\Lambda, \nu}^{a}-g^{-1} c_{\Lambda} V-c_{\Lambda} \mathcal{B}_{(p)_{l},(q)_{m}}^{1}\right) f_{(p)_{l},(q)_{m}-}\right. \\
-c_{\Lambda} \sum_{i=1}^{m} v_{q_{i}} f\left(p_{(p)_{l},(\stackrel{i}{q})_{m}}\right] \|_{V}^{\prime}
\end{gathered}
$$

ISSN 1027-3190. Укр. мат. журн., 2008, m. 60, № 9 
tend to zero in the thermodynamic limit (as $V \rightarrow \infty$ ) for arbitrary fixed numbers $l$ and $m$.

Note that the numbers $l$ and $m$ can tend to infinity together with $V$ but in such a way that $(l+m) / V$ tends to zero as $V \rightarrow \infty$. In this sense the states $f_{(p)_{l},(q)_{m}}$ are the eigenvectors of the Hamiltonian $H_{\Lambda}$ in the thermodynamic limit. If $m=0$, then the states $f(p)_{l}$ are the eigenvectors of the Hamiltonian $H_{\Lambda}$ in the following sense:

$$
\lim _{V \rightarrow \infty}\left\|\left(H_{\Lambda}-H_{\Lambda, \nu}^{a}-c_{\Lambda} \sum_{p} v_{p} a_{-p} a_{p}\right) f_{(p)_{l}}\right\|=0 .
$$

In this case the operator $c_{\Lambda} \sum_{p} v_{p} a_{-p} a_{p}$ on the state $f_{(p)_{l}}$ can be considered as the operator of multiplication in the thermodynamic limit

$$
c_{\Lambda} \sum_{p} v_{p} a_{-p} a_{p} f_{(p)_{l}} \rightarrow V \rightarrow \infty V g^{-1} c_{\Lambda}^{2} f_{(p)_{l}}, \quad \lim _{V \rightarrow \infty} c_{\Lambda}=c .
$$

It follows from the relation

$$
\lim _{V \rightarrow \infty} \frac{1}{V} \sum_{\substack{p \neq(k)_{m} \\ p \neq(p)_{l}}} v_{p} f_{0}^{a}(p)=\int v(p) f_{0}^{a}(p) d p=c .
$$

Thus we use the factor $\frac{1}{V}$ in expression (4.5) mainly to neglect the operator $\left.c_{\Lambda} \sum_{i=1}^{m} v_{q_{i}} f(p)_{l}, \stackrel{i}{\stackrel{i}{q}}\right)_{m}$

Now we give the proof of formula (1). The expression $c_{\Lambda} \sum_{k} v_{k} a_{-k} a_{k} \Phi_{0}$ can be estimated as follows: The sum

$$
\begin{gathered}
\mathcal{A}^{-} \Phi_{0}=c_{\Lambda} \sum_{k} v_{k} a_{-k} a_{k} \Phi_{0}=c_{\Lambda} \sum_{k} v_{k} f_{1}^{0}(k)|0\rangle+ \\
+c_{\Lambda} \sum_{n=2}^{\infty} \frac{1}{n !} \sum_{i=1}^{n} \sum_{k_{1}} f_{1}^{0}\left(k_{1}\right) a_{k_{1}}^{+} a_{-k_{1}}^{+} \ldots \sum_{\substack{k_{i} \neq k_{1} \\
\vdots \\
k_{i} \neq k_{n}}} v_{k_{i}} f_{1}^{0}\left(k_{i}\right) \ldots \sum_{k_{n}} f_{1}^{0}\left(k_{n}\right) a_{k_{n}}^{+} a_{-k_{n}}^{+}|0\rangle,
\end{gathered}
$$

should be divided into two parts $0 \leq n \leq n_{0}$ and $n_{0}<n<\infty$, and the number $n_{0}$ is of order $V^{\delta}$, where $0<\delta<1$, i.e., $n_{0} \leq V^{\delta}$. Then one has

$$
\begin{aligned}
\lim _{V \rightarrow \infty} \frac{1}{V^{2}}\left(\mathcal{A}^{-} \Phi_{0}, \mathcal{A}^{-} \Phi_{0}\right)^{\prime}=\lim _{V \rightarrow \infty} \frac{1}{V^{2}} c_{\Lambda}^{2}\left(\sum_{k} v_{k} f_{1}^{0}(k)\right)^{2}+ \\
+\lim _{V \rightarrow \infty} c_{\Lambda}^{2} \sum_{n=2}^{n_{0}} \frac{1}{V^{n-1}} \sum_{k_{1} \neq \ldots \neq k_{n}}^{\prime}\left|f_{1}^{0}\left(k_{1}\right)\right|^{2} \ldots \stackrel{i}{V} \ldots\left|f_{1}^{0}\left(k_{n}\right)\right|^{2} \times \\
\times\left(\frac{1}{V} \sum_{k_{i} \neq k_{1}, \ldots, k_{i} \neq k_{n}} v_{k_{i}} f_{1}^{0}\left(k_{i}\right)\right)^{2}+
\end{aligned}
$$




$$
\begin{gathered}
+\lim _{V \rightarrow \infty} c_{\Lambda}^{2} \sum_{n=n_{0}+1}^{\infty} \frac{1}{V^{n-1}} \sum_{k_{1} \neq \ldots \neq k_{n}}^{\prime}\left|f_{1}^{0}\left(k_{1}\right)\right|^{2} \ldots \stackrel{i}{\vee} \ldots\left|f_{1}^{0}\left(k_{n}\right)\right|^{2} \times \\
\times\left(\frac{1}{V} \sum_{k_{i} \neq k_{1}, \ldots, k_{i} \neq k_{n}} v_{k_{i}} f_{1}^{0}\left(k_{i}\right)\right)^{2} .
\end{gathered}
$$

For $n \leq n_{0}$ one has

$$
\begin{gathered}
\lim _{V \rightarrow \infty} \frac{1}{V} \sum_{k_{i} \neq k_{1}, \ldots, k_{i} \neq k_{n}} v_{k_{i}} f_{1}^{0}\left(k_{i}\right)= \\
=\lim _{V \rightarrow \infty} \frac{1}{V}\left(\sum_{(k)_{i}} v_{k_{i}} f_{1}^{0}\left(k_{i}\right)-\sum_{k_{i} \neq k_{1}, \ldots, k_{i} \neq k_{n}} v_{k_{i}} f_{1}^{0}\left(k_{i}\right)\right)= \\
=\lim _{V \rightarrow \infty} \frac{1}{V} \sum_{k_{i}} v_{k_{i}} f_{1}^{0}\left(k_{i}\right)=\int v\left(k_{i}\right) f_{1}^{0}\left(k_{i}\right) d k_{i}=c,
\end{gathered}
$$

because

$$
\lim _{V \rightarrow \infty} \frac{1}{V}\left|\sum_{k_{i} \neq k_{1}, \ldots, k_{i} \neq k_{n}} v_{k_{i}} f_{1}^{0}\left(k_{i}\right)\right| \leq \frac{1 n}{V} v f \leq \lim _{V \rightarrow \infty} \frac{V^{\delta}}{V} v f=0
$$

for $n<n_{0}<V^{\delta}, 0<\delta<1$. For the sum over $n \geq n_{0}+1$ one has the following estimate:

$$
\begin{gathered}
\sum_{n=n_{0}+1}^{N} \frac{1}{V^{n-1}} \sum_{k_{1} \neq \ldots \neq k_{n}}^{\prime}\left|f_{1}^{0}\left(k_{1}\right)\right|^{2} \ldots \vee^{i} \ldots\left|f_{1}^{0}\left(k_{n}\right)\right|^{2}\left|\frac{1}{V} \sum_{k_{i} \neq k_{1}, \ldots, k_{i} \neq k_{n}} v_{k_{i}} f_{1}^{0}\left(k_{i}\right)\right|^{2} \leq \\
\leq \sum_{n=n_{0}+1}^{N} \frac{1}{V^{n-1}} \frac{N^{n-1}}{(n-1) !} f^{2 n} v^{2} n^{2} \leq \sum_{n=n_{0}+1}^{\infty} \alpha^{n-1} f^{2 n} v^{2} \frac{n^{2}}{(n-1) !} .
\end{gathered}
$$

The last series is convergent and tends to zero as $V \rightarrow \infty$ because $n_{0}$. Thus,

$$
\lim _{V \rightarrow \infty} \frac{1}{V^{2}}\left(A^{-} \Phi_{0}, A^{-} \Phi_{0}\right)_{V}^{\prime}=g^{-2} c^{4} \lim _{V \rightarrow \infty}\left(\Phi_{0}, \Phi_{0}\right)_{V}^{\prime}
$$

where $\lim _{V \rightarrow \infty}\left(\Phi_{0}, \Phi_{0}\right)_{V}^{\prime}=\sum_{n=0}^{\infty} \frac{1}{n !} \sum_{k_{1} \neq \ldots \neq k_{n}}\left|f_{0}^{a}\left(k_{1}\right)\right|^{2} \ldots \stackrel{i}{\vee} \ldots\left|f_{0}^{a}\left(k_{n}\right)\right|^{2}=$ $=e^{\int\left|f_{0}^{a}(k)\right|^{2} d k}$. Using an analogous calculation one can show that

$$
\lim _{V \rightarrow \infty} \frac{1}{V}\left(\Phi, A^{-} \Phi_{0}\right)_{V}^{\prime}=g^{-1} c^{2} \lim _{V \rightarrow \infty}\left(\Phi, \Phi_{0}\right)_{V}^{\prime}
$$

for an arbitrary coherent state

$$
\Phi=\exp \left(\sum_{k} f_{k} a_{k}^{+} a_{-k}^{+}\right)|0\rangle, \quad\left|f_{k}\right|<f<\infty .
$$

ISSN 1027-3190.Укр. мат. журн., 2008, m. 60, № 9 
In the above described sense the operator $\frac{1}{V} A^{-}$on $\Phi_{0}$ in the thermodynamic limit is equal to the operator of multiplication by constant $g^{-1} c^{2}$.

By analogous calculation one can show that

$$
\begin{gathered}
\lim _{V \rightarrow \infty} c_{\Lambda} \sum_{p} v_{p} a_{-p} a_{p} \Phi_{(p)_{l},(q)_{m}}= \\
=c_{\Lambda} \lim _{V \rightarrow \infty}\left[a_{p_{1}}^{+} \ldots a_{p_{l}}^{+} a_{q_{1}}^{+} a_{-q_{1}}^{+} \ldots a_{q_{m}}^{+} a_{-q_{m}}^{+} \sum_{n=0}^{\infty} \frac{1}{n !} \sum_{k_{1}} f_{0}^{a}\left(k_{1}\right) a_{k_{1}}^{+} a_{-k_{1}}^{+} \ldots\right. \\
\left.\ldots \sum_{k_{n}} f_{0}^{a}\left(k_{n}\right) a_{k_{n}}^{+} a_{-k_{n}}^{+}|0\rangle \sum_{k \neq(k)_{n}, k \neq(p)_{l}, k \neq(q)_{m}} v_{k} f_{0}^{a}(k)+c_{\Lambda} \sum_{i=1}^{m} v_{q_{i}} \Phi{ }_{(p)_{l},(\stackrel{i}{q})_{m}}\right]= \\
=g^{-1} c^{2} V \Phi_{(p)_{l},(q)_{m}}+c \sum_{i=1}^{m} v_{q_{i}} \Phi \\
(p)_{l},(\stackrel{i}{v})_{m}
\end{gathered}
$$

Remark 4.1. The factor $\frac{1}{V}$ in expressions (4.5) could be replaced by factor $\frac{1}{V^{\delta}}$ where $\delta$ is arbitrary number $0<\delta<1$ for arbitrary fixed $m$.

Remark 4.2. Consider the excitation created by the operators $\alpha_{k}^{+} \alpha_{-k}^{+}$or $\alpha_{k} \alpha_{-k}$. It is easy to check that

$$
\begin{gathered}
\alpha_{p}^{+} \Phi_{0}=\sqrt{1+\left(f^{a}(p)\right)^{2}} a_{p}^{+} \prod_{k \neq p}\left(1+f^{a}(k) a_{k}^{+} a_{-k}^{+}\right)|0\rangle, \\
\alpha_{-p}^{+} \Phi_{0}=\sqrt{1+\left(f^{a}(p)\right)^{2}} a_{-p}^{+} \prod_{k \neq p}\left(1+f^{a}(k) a_{k}^{+} a_{-k}^{+}\right)|0\rangle, \\
\alpha_{q}^{+} \alpha_{-q}^{+} \Phi_{0}=\left(-f^{a}(q)+a_{q}^{+} a_{-q}^{+}\right) \prod_{k \neq q}\left(1+f^{a}(k) a_{k}^{+} a_{-k}^{+}\right)|0\rangle .
\end{gathered}
$$

It follows from the obtained formulas that the excitations

$$
\varphi_{(p)_{l},(q)_{m}}=\alpha_{p_{1}}^{+} \ldots \alpha_{p_{l}}^{+} \alpha_{q_{1}}^{+} \alpha_{-q_{1}}^{+} \ldots \alpha_{q_{m}}^{+} \alpha_{-q_{m}}^{+} \Phi_{0}^{a}
$$

can be expressed via certain linear combinations of excitations $f_{(p)_{l},(q)_{i}}, i=0,1, \ldots, m$, where expressions $\sqrt{1+\left(f^{a}\left(p_{i}\right)\right)^{2}}, i=1, \ldots, l, f^{a}\left(q_{j}\right), j=1, \ldots, m$, should be considered as coefficients independent on momenta $(k)_{n}$. All results obtained above about coincidence in the thermodynamic limit of the model BCS and approximating Hamiltonians on the excitations $f_{(p)_{l},(q)_{m}}$ also hold for the excitations $\varphi_{(p)_{l},(q)_{m}}$.

1. Petrina D. Ya. On Hamiltonians of quantum statistics and a model Hamiltonian in the theory of superconductivity // Teor. i Mat. Fiz. - 1970. - 4, № 3. - P. 394-411.

2. Petrina D. Ya., Yatsyshin V. P. On model Hamiltonian in the theory of superconductivity // Ibid. 1972. - 10, № 2. - P. $283-299$.

3. Petrina D. Ya. Spectrum and states of BCS Hamiltonian in finite domain. I. Spectrum // Ukr. Mat Zh. - 2000. - 52, № 5. - P. $667-690$.

4. Petrina D. Ya. Spectrum and states of the BCS Hamiltonian in finite domains. II. Spectra of excitations // Ibid. -2001 . - 53, № 8. - P. 1080-1101. 
5. Petrina D. Ya. Spectrum and states of the BCS Hamiltonian in finite domain. III. The BCS Hamiltonian with mean-field interaction // Ibid. - 2002. - 54, № 11. - P. $1486-1504$.

6. Petrina D. Ya. Model BCS Hamiltonian and approximating Hamiltonian for an infinite volume. IV. Two branches of their common spectra and states // Ibid. - 2003. - 55, № 2. - P. 174-197.

7. Petrina D. Ya. New second branch of spectrum of the BCS Hamiltonian and "pseudo-gap" // Ibid. - 2005. - 57, № 11. - P. $1508-1534$.

8. Petrina D. Ya. Mathematical foundations of quantum statistical mechanics. - Dordrecht: Kluwer Acad. Publ., 1995. - 461 p.

9. Bardeen J., Cooper L. N., Schrieffer J. R. Theory of superconductivity // Phys. Rev. - 1957. - 108. - P. $1175-1204$.

10. Cooper L. N. Bound electron pairs in a degenerate Fermi gas // Ibid. - 1956. - 104. - P. 1189-1190.

11. Bogolyubov N. N. On the model Hamiltonian in the theory of superconductivity // Selected Paper of N. N. Bogolyubov. - Kiev: Naukova Dumka, 1970. - 3. - P. 110-173.

12. Bogolyubov N. N. (jr.) A method of investigations of model Hamiltonian. - Moscow: Nauka, 1974 $-178 \mathrm{p}$.

13. Bogolyubov N. N., Zubarev D. N., Tserkovnikov Yu. A. Asymptotically exact solution for model Hamiltonian of theory of superconductivity // Selected Papers of N. N. Bogolyubov. - Kiev: Naukova Dumka, 1960. - 3. - P. $98-109$.

Received 28.04.07 